\title{
Differential Implication of Proinflammatory Cytokine Interleukin-6 in the Development of Cephalic versus Extracephalic Neuropathic Pain in Rats
}

\author{
Alban Latrémolière, ${ }^{1,2}$ Annie Mauborgne, ${ }^{1,3}$ Justine Masson, ${ }^{1,2}$ Sylvie Bourgoin, ${ }^{1,2}$ Valérie Kayser, ${ }^{1,2}$ Michel Hamon, ${ }^{1,2}$ \\ and Michel Pohl 1,3 \\ ${ }^{1}$ Université Pierre et Marie Curie-Paris 6, Faculté de Médecine Pierre et Marie Curie, Site Pitié-Salpêtrière, Institut Fédératif de Recherche 70 des \\ Neurosciences, Unité Mixte de Recherche S677, F-75013 Paris, France, and ${ }^{2}$ Inserm, Unité 677 and ${ }^{3}$ Unité 713, F-75013 Paris, France
}

\begin{abstract}
Responses resulting from injury to the trigeminal nerve exhibit differences compared with those caused by lesion of other peripheral nerves. With the aim of elucidating the physiopathological mechanisms underlying cephalic versus extracephalic neuropathic pain, we determined the time course expression of proinflammatory cytokines interleukin-6 (IL-6) and IL-1 $\beta$, neuronal injury (ATF3), macrophage/microglial (OX-42), and satellite cells/astrocyte (GFAP) markers in central and ganglion tissues in rats that underwent unilateral chronic constriction injury (CCI) to either infraorbital nerve (IoN) (cephalic area) or sciatic nerve (SN) (extracephalic area). Whereas CCI induced microglial activation in both models, we observed a concomitant upregulation of IL-6 and ATF3 in the ipsilateral dorsal horn of the lumbar cord in SN-CCI rats but not in the ipsilateral spinal nucleus of the trigeminal nerve ( $\mathrm{Sp} 5 \mathrm{c})$ in IoN-CCI rats. Preemptive treatment with minocycline (daily administration of $20 \mathrm{mg} / \mathrm{kg}$, i.p., for 2 weeks) partially prevented pain behavior and microglial activation in SN-CCI rats but was ineffective in IoN-CCI rats. We show that IL-6 can upregulate 0X-42 and ATF3 expression in cultured microglia and neurons from spinal cord, respectively, as well as in the dorsal horn after acute intrathecal administration of the cytokine. We propose that IL- 6 could be one of the promoters of the signaling cascade leading to abnormal pain behavior in SN-CCI but not IoN-CCI rats. Our data further support the idea that different pathophysiological mechanisms contribute to the development of cephalic versus extracephalic neuropathic pain.
\end{abstract}

Key words: neuropathic pain; trigeminal; microglia; minocycline; IL-6; ATF3

\section{Introduction}

Neuropathic pain caused by peripheral nerve injury is characterized by long-lasting exaggerated pain behavior such as hyperalgesia and allodynia. Numerous studies have revealed differences between the pain syndrome caused by injury to the trigeminal nerve and those occurring after lesion of other peripheral nerves (Tal and Devor, 1992; Benoliel et al., 2001; Kayser et al., 2002), suggesting the existence of distinct pathophysiological mechanisms in the cephalic versus extracephalic territories (Bennett, 2004). Precise pathophysiological mechanisms implicated in the development of neuropathic pain states are still unclear, but numerous data have recently suggested a major role for activated glial cells via the production of cytokines (Watkins et al., 2001). Indeed, proinflammatory cytokines [such as interleukin-6 (IL-6)

\footnotetext{
Received June 4, 2008; revised July 2, 2008; accepted July 6, 2008.

This work was supported by grants from Inserm and Université Pierre et Marie Curie-Paris 6. A.L. was a recipient of fellowships from the French Ministère de la Recherche et de l'Enseignement Supérieur, Agence Nationale de la Recherche, and Fondation pour la Recherche Médicale during performance of this work. We are grateful to Dr. Chloé Alexandre for her help with statistical analyses.

Correspondence should be addressed to Dr. Alban Latrémolière, Unité Mixte de Recherche 677, Inserm/Université Pierre et Marie Curie, Faculté de Médecine Pierre et Marie Curie, Site Pitié-Salpêtrière, 75634 Paris Cedex 13, France. E-mail: allodynie@hotmail.com.

DOI:10.1523/JNEUROSCI.2552-08.2008

Copyright $\odot 2008$ Society for Neuroscience $\quad$ 0270-6474/08/288489-13\$15.00/0
}

and IL-1 $\beta$ ] have been reported to be upregulated in ipsilateral ganglia (Murphy et al., 1995; Lee et al., 2004) and spinal cord (Arruda et al., 1998; Raghavendra et al., 2002, 2003; Lee et al., 2004) after nerve injury. Moreover, both the overexpression of these cytokines and the associated pain behavior could be prevented in injured animals by minocycline, a semisynthetic second-generation tetracycline described as an inhibitor of microglial activation in animal models of neuropathic pain (Raghavendra et al., 2003; Ledeboer et al., 2005; Piao et al., 2006; Scholz and Woolf, 2007), further accrediting the idea that this cell type plays a key role in neuropathic pain induction. However, upregulation of activating transcription factor 3 (ATF3), a marker of neuronal damage/injury (Tsujino et al., 2000; Hai and Hartman, 2001), is also associated with the establishment of longlasting pain conditions (Obata et al., 2003; Shortland et al., 2006), indicating that neuronal injury is also involved in neuropathic pain.

In this study, we sought to investigate the implication of proinflammatory cytokines and to assess how interactions between injured neurons and surrounding glial cells can participate in the development of persistent pain states in two animal models of nerve injury at the cephalic versus extracephalic level. We first determined the time course changes in the levels of mRNAs encoding markers of nerve alteration and/or glial activation, such as 
IL-6, IL-1 $\beta$, ATF3, OX-42 (integrin $\alpha$ M, a marker of macrophages/microglia) and glial fibrillary acidic protein (GFAP) (a marker of satellite cells/astrocytes) in sensory ganglia and central tissues after chronic constriction injury (CCI) to either infraorbital nerve (IoN) (cephalic area) or sciatic nerve (SN) (extracephalic area). We then assessed the putative anti-allodynic effects of minocycline in both models by using a preventive paradigm. The distinct effects of this drug depending on the nerve injury model strongly support the idea that specific cascades of events are implicated in the development of cephalic versus extracephalic neuropathic pain.

\section{Materials and Methods \\ Animals}

All experiments were performed in conformity with institutional guidelines, which are in compliance with national and international laws and policies for use of animals in neuroscience research [European Communities Council Directive number 87848, October 1987, Ministère de l'Agriculture et de la Forêt, Service Vétérinaire de la Santé et de la Protection Animale; permissions numbers 006228 (to S.B.), 00482 (to V.K.), 75-116 (to M.H.), and 00186 (to M.P.)]. Male Sprague Dawley rats (Charles River Laboratories), weighing 150-200 g on arrival, were used. In addition, pregnant females at gestation day 15 were received from the same breeding center. All animals were maintained under the same conditions $\left(22 \pm 1{ }^{\circ} \mathrm{C}, 60 \%\right.$ relative humidity, $12 \mathrm{~h}$ light/dark cycle, food and water ad libitum). Animals were accustomed to the housing facilities for at least 1 week before any treatment.

\section{Chronic constriction injury to the infraorbital nerve}

Rats (150-175 g) were anesthetized with an intraperitoneal injection of sodium pentobarbital (Nembutal, $50 \mathrm{mg} / \mathrm{kg}$ ). Unilateral chronic constriction injury to the infraorbital nerve was performed under direct visual control using a Zeiss microscope $(10-25 \times)$ essentially as described by Vos et al. (1994). Briefly, the head was fixed in a HorsleyClarke stereotaxic frame, and a midline scalp incision was made, exposing skull and nasal bone. The edge of the orbit, formed by the maxillary, frontal, lacrimal, and zygomatic bones, was dissected free. The orbital contents were gently deflected to give access to the infraorbital nerve, which was dissected free at its most rostral extent in the orbital cavity, just caudal to the infraorbital foramen. Only $5 \mathrm{~mm}$ of the nerve could be freed (Vos et al., 1994), providing the space for placement of two chromic catgut (5-0) ligations tied loosely (with $\sim 2 \mathrm{~mm}$ spacing) around it. To obtain the desired degree of constriction, the criterion formulated by Bennett and Xie (1988) was used: the ligations reduced the diameter of the nerve by a just noticeable amount and retarded, but did not interrupt, the epineural circulation. Blood circulation through epineural vessels was checked under direct visual control using the Zeiss operation microscope. Finally, scalp incision was closed using silk sutures (4-0). In shamoperated rats, the IoN was exposed using the same procedure but was not ligated.

\section{Chronic constriction injury to the sciatic nerve}

Rats (175-200 g) were anesthetized with sodium pentobarbital (Nembutal, $50 \mathrm{mg} / \mathrm{kg}$, i.p.), and the common sciatic nerve was exposed. Using a dissection microscope ( $2 \times$ magnification), four chromic catgut (5-0) ligations were tied loosely with $\sim 1 \mathrm{~mm}$ spacing, proximally to the sciatic trifurcation (Bennett and Xie, 1988). Finally, the skin and muscle were sewed using silk sutures (4-0). In sham-operated animals, the same surgery was performed, but the nerve was not ligated.

For both IoN-CCI and SN-CCI surgeries, rats recovered from anesthesia in a postoperative chamber on a warming pad and then returned to their home cages (four animals per cage).

\section{Pharmacological studies}

In a systemic preventive paradigm, minocycline (Sigma-Aldrich) at 20 $\mathrm{mg} / \mathrm{kg}$ (Mino20) or $40 \mathrm{mg} / \mathrm{kg}$ (Mino40) or sterile water (vehicle) was administered intraperitoneally $1 \mathrm{~h}$ before IoN-CCI or SN-CCI and then once daily (between 3:00 P.M. and 5:00 P.M.) for the following 14 d. On day 14, allodynia-like behavior was assessed, and animals were killed for biochemical analyses.

\section{Behavioral testing}

All behavioral assessments were conducted between 9:00 A.M. and 5:00

P.M. in a quiet room.

In IoN-CCI rats. Nociceptive behavioral tests were performed one day before and then $6 \mathrm{~h}$ [day $0.25(\mathrm{~d} 0.25)$ ] and $1 \mathrm{~d}(\mathrm{~d} 1), 7 \mathrm{~d}(\mathrm{~d} 7), 14 \mathrm{~d}(\mathrm{~d} 14)$, $21 \mathrm{~d}(\mathrm{~d} 21)$, or $140 \mathrm{~d}(\mathrm{~d} 140)$ after completion of surgery. The rats were placed individually in small $(35 \times 20 \times 15 \mathrm{~cm})$ plastic cages. Before any stimulation session, rats freely explored the observation cage and the testing environments for $2 \mathrm{~h}$. During this habituation period, the experimenter reached slowly into the cage to touch its walls with a plastic rod, similar to the one on which the von Frey filaments are mounted. After $2 \mathrm{~h}$, rats were generally in a sniffing/no locomotion state (with the four paws on the ground, neither moving nor freezing), and the stimulation session could start. In rare cases, a rat could still be moving/exploring after $2 \mathrm{~h}$, and the habituation period was then prolonged to $3 \mathrm{~h}$. Mechanical sensitivity was determined with a graded series of 11 von Frey filaments (Bioseb). The filaments produced a bending force of $0.07,0.16$, $0.40,0.60,1.00,2.00,4.00,6.00,8.00,10.00$, and $12.00 \mathrm{~g}$, respectively. The stimuli were applied within the IoN territory (vibrissae pad) three times on the nerve-injured side and then on the contralateral side for a total of six applications of each filament per rat, always beginning with the filament producing the lowest force. The von Frey filaments were applied at least $3 \mathrm{~s}$ after the rats had returned to their initial resting state. For each session, the complete series of von Frey filaments were tested in increasing force order. Behavioral nociceptive response consisted of (1) a brisk withdrawal reaction: the rat pulls briskly backward; (2) an escape/attack: the rat avoids additional contact with the filament either passively by moving its body away from the stimulating object to assume a crouching position against cage wall, sometimes with the head buried under the body, or actively by attacking the stimulating object, making biting and grabbing movements; or (3) asymmetric face grooming: the rat displays an uninterrupted series of at least three face-wash strokes directed to the stimulated facial area, often preceded by the brisk withdrawal reaction. The latter responses represent the highest scores in the rank-ordered response scoring system initially described by Vos et al. (1994). The minimal force filament causing at least one among these responses (to at least two of the three on each side) allowed determination of the mechanical response threshold. The $12.00 \mathrm{~g}$ filament was chosen as the cutoff threshold to prevent tissue injury. In the preoperative tests, stimulation with the $12.00 \mathrm{~g}$ filament did not induce any nociceptive behavior in the majority of the rats $(>90 \%)$. To avoid nonspecific responses, only these rats were included in the study.

In $\mathrm{SN}$-CCI rats. Before testing, each rat was habituated in a small plastic $(35 \times 20 \times 15 \mathrm{~cm})$ cage for $2 \mathrm{~h}$. Then, as for the IoN-CCI rats, mechanical sensitivity was determined with a graded series of eight von Frey filaments that produced a bending force of 2, 4, 6, 8, 10, 12, 26, and $60 \mathrm{~g}$, respectively. The stimuli were applied within the SN territory (lateral part of the hindpaw). Each filament was tested three times on the nerve-injured side and then on the contralateral side for a total of six applications per rat in increasing order starting with the filament producing the lowest force. As for the IoN-CCI rats, the von Frey filaments were applied at least $3 \mathrm{~s}$ after the rats had returned to their initial resting state. The minimal force filament for which animals presented either a brisk paw withdrawal and/or an escape attempt in response to at least two of the three stimulations allowed determination of the mechanical response threshold.

\section{Tissue collection and RNA extraction}

Animals used for reverse transcription (RT)-PCR or ELISA procedures were killed by decapitation at d0.25, d1, d7, d14, d21, or d140 after surgery depending on the experiment. Trigeminal ganglia (TG) and spinal nucleus pars caudalis (Sp5c) for IoN-CCI and L4-L5 dorsal root ganglia (DRG) and the ipsilateral and contralateral dorsal quadrants of the lumbar enlargement of the spinal cord for SN-CCI were dissected at $0-4^{\circ} \mathrm{C}$. Tissue pieces for RNA analyses were immediately frozen in liquid nitrogen and stored at $-80^{\circ} \mathrm{C}$. Total RNA was extracted using the Nucle- 
oSpin RNA II extraction kit (Macherey-Nagel) and quantified from optical density at $260 \mathrm{~nm}$ using as reference a scale of total RNA prepared on a cesium chloride gradient.

\section{Real time RT-PCR}

First-stranded cDNA synthesis (from 660 ng of total RNA per $20 \mu \mathrm{l}$ reaction mixture) was performed using SuperScript III Reverse Transcriptase and random primers ( $250 \mathrm{ng}$ per reaction), as recommended by the manufacturer (Invitrogen). PCR amplification, in triplicate for each sample, was performed using ABI Prism 7300 (Applied Biosystems), ABsolute QPCR ROX Mix (ABgene), and Assays-on-Demand Gene Expression probes (Applied Biosystems) for target genes: IL-6 (assay ID Rn00561420_m1), IL-1 $\beta$ (Rn00580432_m1), GFAP (Rn01460868_m1), OX-42 (Rn00709342_m1), and ATF3 (Rn00563784_m1). Semiquantitative determinations were made with reference to reporter genes encoding glyceraldehyde 3-phosphate dehydrogenase (GaPDH) (Rn99999916_s1) and ribosomal subunit 18S (Hs99999901_s1). Because the relative expression of GaPDH compared with $18 \mathrm{~S}$ was not significantly different at $6 \mathrm{~h}$ and $14 \mathrm{~d}$ after injury, most of the experiments were performed with $\mathrm{GaPDH}$ only as reporter gene. The polymerase activation step at $95^{\circ} \mathrm{C}$ for $15 \mathrm{~min}$ was followed by 40 cycles of $15 \mathrm{~s}$ at $95^{\circ} \mathrm{C}$ and $60 \mathrm{~s}$ at $60^{\circ} \mathrm{C}$. The validity of the results was checked by running appropriate negative controls (replacement of cDNA by water for PCR amplification; omission of reverse transcriptase for cDNA synthesis). Specific mRNA levels were calculated after normalizing from GaDPH mRNA in each sample. Data are presented as relative mRNA units compared with control values (expressed as fold over sham value).

\section{Immunohistochemistry}

Deeply anesthetized animals (sodium pentobarbital, $50 \mathrm{mg} / \mathrm{kg}$, i.p.) were perfused intracardially with $100 \mathrm{ml}$ of saline $(0.9 \% \mathrm{NaCl})$ supplemented with $0.1 \%$ sodium nitrite, followed by $600 \mathrm{ml}$ of $4 \%$ paraformaldehyde in PBS at room temperature. TG and Sp5c for IoN-CCI and DRG and L4-L5 dorsal quadrants for SN-CCI were dissected out and cryoprotected in $10 \%$ sucrose $\left(24 \mathrm{~h}, 4^{\circ} \mathrm{C}\right)$. Fifteen micrometer sections were cut in a cryostat $\left(-20^{\circ} \mathrm{C}\right)$ and preincubated $(30 \mathrm{~min}$, room temperature) in PBS containing $0.3 \%$ Triton X-100 and 3\% normal donkey serum (Interchim). Sections were then incubated overnight at $4^{\circ} \mathrm{C}$ in the same buffer supplemented with a mouse monoclonal anti-OX-42 antibody (1:100; Serotec) or a rabbit polyclonal anti-ATF3 antibody (1:200; Santa Cruz Biotechnology). After washing in PBS, sections were incubated for $1 \mathrm{~h}$ with rhodamine [cyanine 5 (Cy5)]-conjugated anti-rabbit Ig or rhodamine (Cy3)-conjugated anti-mouse Ig (1:800; Interchim), rinsed in PBS, mounted in Fluoromount-G (Clinisciences), and examined using a Leica confocal microscope.

\section{ELISA quantification of IL-6}

Tissues were weighed and homogenized by sonication in a lysis buffer $(50$ $\mathrm{mg}$ of tissue in $250 \mu \mathrm{l}$ ) containing $50 \mathrm{~mm} \mathrm{NaCl}, 10 \mathrm{~mm}$ Tris, $2.5 \mathrm{~mm}$ $\mathrm{MgCl}_{2}$, and the protease inhibitor cocktail 2 (Sigma-Aldrich) at $\mathrm{pH}$ 7.4. Sonicated samples were centrifuged at $20,000 \times g$ for $30 \mathrm{~min}$ at $4^{\circ} \mathrm{C}$, and supernatants were aliquoted and stored at $-80^{\circ} \mathrm{C}$ until protein quantification. IL-6 protein concentrations were determined using the quantitative sandwich enzyme immunoassay ( $\mathrm{R} \& \mathrm{D}$ Systems) according to the protocol of the manufacturer. Data are expressed in picograms of IL-6 per milligram of tissue.

\section{Cell culture and immunocytofluorescence}

Primary culture of microglia. Primary cultures of microglia were prepared using the shaking method as described previously (Krady et al., 2002; Salimi and Humpel, 2002), with few modifications. Briefly, midbrain and spinal cord of 13-14 rat pups at postnatal day 1 were freed from meninges and then dissociated in HBSS containing $50 \mathrm{~mm}$ HEPES. Tissue suspension was centrifuged $(400 \times g ; 30 \mathrm{~s})$, and the pellet was resuspended in 10 $\mathrm{ml}$ of HBSS containing $50 \mathrm{~mm}$ HEPES, $0.25 \%(\mathrm{w} / \mathrm{v})$ trypsin, and 0.2 $\mathrm{mg} / \mathrm{ml}$ Dnase I and then incubated $15 \mathrm{~min}$ at $37^{\circ} \mathrm{C}$. After addition of $5 \%$ fetal calf serum (final dilution), the dissociated cell suspension was centrifuged $(400 \times g ; 10 \mathrm{~min}$ ), and the pellet was resuspended in $50 \mathrm{ml}$ of DMEM supplemented with $10 \%$ heat-inactivated fetal calf serum and $1 \%(\mathrm{w} / \mathrm{v})$ penicillin/streptomycin (complete culture medium). The sus- pension was centrifuged as above until a clear supernatant was obtained (one to two rounds of centrifugation), and the final pellet was resuspended in complete culture medium for seeding at the density of $5 \times 10^{4}$ cells $/ \mathrm{cm}^{2}$ in $58 \mathrm{~cm}^{2}$ sterile Petri dishes (Costar, VWR International). The culture medium was changed at $1 \mathrm{~d}$ in vitro (DiV1) and DiV3. On DiV10, the dishes were gently shaken $(300 \mathrm{rpm}$ ) on a platform shaker for $30 \mathrm{~min}$, and each dish content was harvested and centrifuged for $10 \mathrm{~min}$ at $1000 \times g$ at $4^{\circ} \mathrm{C}$. Microglia cells in the pellet were resuspended in complete culture medium and distributed in six-well plates at the density of $3 \times 10^{5}$ cells per well. IL-6 (1-100 ng/ml; Sigma-Aldrich) and/or minocycline hydrochloride ( $100 \mu \mathrm{M}$; Sigma-Aldrich) were added into the culture medium, and cells were further incubated for 1.5 or $6 \mathrm{~h}$, before processing for RT-PCR or immunocytochemical experiments. The purity of isolated microglia was assessed by immunocytochemical staining for OX-42 and GFAP. Under these conditions, $>95 \%$ of cells in primary culture expressed OX-42 but not GFAP (data not shown).

Primary culture of spinal cord neurons. Primary cultures of mixed spinal cord neurons/glia were performed as described by Goslin et al. (1998) with some modifications. Spinal cord was dissected at embryonic day 14 . Tissues from 10 rat embryos were incubated (Neurobasal, $10 \mathrm{ml}$ ) with trypsin $\left(0.25 \% ; 10 \mathrm{~min}\right.$ at $\left.37^{\circ} \mathrm{C}\right)$, and dissociation was achieved by five successive passages through a Pasteur pipette. Cells were counted and plated either on poly-L-lysine-coated four-chamber slides at a density of 75,000 cells per well (for immunocytochemistry) or on six-well plates for RT-PCR experiments (at a density of 300,000 cells per well), in complete Neurobasal medium supplemented with B27 (Invitrogen), $1 \mathrm{~mm}$ L-glutamine, penicillin $\mathrm{G}(10 \mathrm{U} / \mathrm{ml})$, and streptomycin $(10 \mathrm{mg} / \mathrm{ml})$. Four hours after plating, the coverslips were either transferred to a confluent plate of glial cells and maintained for $24 \mathrm{~h}$ in complete conditioned Neurobasal medium (mixed glia/neurons primary culture) or transferred and maintained in complete conditioned Neurobasal medium (enriched neurons primary culture). Three days after plating, IL-6 and/or minocycline hydrochloride were added into the culture medium, and cells were further incubated during 1.5 or $6 \mathrm{~h}$, before RT-PCR or immunocytofluorescence experiments.

\section{Immunocytofluorescence}

Coverslips were washed twice with PBS containing $0.1 \mathrm{mM} \mathrm{CaCl}_{2}$ and 0.1 $\mathrm{mM} \mathrm{MgCl}_{2}$ (PBS+), fixed with $4 \%$ paraformaldehyde for $5 \mathrm{~min}$ in PBS+, and rinsed $(3 \times 10 \mathrm{~min})$ in $\mathrm{PBS}+$. After a $30 \mathrm{~min}$ preincubation in $\mathrm{PBS}$ containing $0.3 \%$ Triton X-100 and 3\% normal donkey serum (Interchim) at room temperature, cells were incubated overnight at $4^{\circ} \mathrm{C}$ in the same buffer supplemented with a rabbit polyclonal anti-ATF3 antibody (1:200; Santa Cruz Biotechnology), a mouse polyclonal anti-GFAP antibody (1:300; DakoCytomation), or a mouse monoclonal anti-neuronalspecific nuclear protein (NeuN) antibody (1:200; Millipore Bioscience Research Reagents). After a three times, 10 min washing in PBS+, coverslips were incubated for $1 \mathrm{~h}$ at room temperature with rhodamine (Cy5)-conjugated anti-rabbit Ig or rhodamine (Cy3)-conjugated antimouse Ig (1:800; Interchim), rinsed in PBS, mounted in Fluoromount-G (Clinisciences), and examined using a Leica confocal microscope.

\section{Statistical analyses}

All values are expressed as means \pm SEM. For behavioral tests, results were analyzed using two-way ANOVA with factor treatment (i.e., IoNCCI or SN-CCI vs respective sham) and repeated measures over time. For RT-PCR data, the $2^{-\Delta \Delta C t}$ method (Livak and Schmittgen, 2001) was used to analyze the relative changes in specific mRNA levels between different groups (RQ Study Software 1.2 version; Applied Biosystems). For the time course expression of the target genes, a two-way ANOVA was performed with factors treatment (i.e., IoN-CCI or SN-CCI vs respective sham) and condition (i.e., postoperative days $\mathrm{d} 0.25-\mathrm{d} 140$ for IoN-CCI rats and d0.25-d21 for SN-CCI rats) (supplemental Table 1, available at www.jneurosci.org as supplemental material). The effects of minocycline treatment on behavior and mRNAs expression were evaluated by a two-way ANOVA with factors treatment (i.e., IoN-CCI or $\mathrm{SN}-\mathrm{CCI}$ vs respective sham) and drug (i.e., minocycline or vehicle) (supplemental Table 2, available at www.jneurosci.org as supplemental material). For ELISA dosage of IL-6, a one-way ANOVA with factor treat- 


\section{A $\quad \mathrm{ION}-\mathrm{CCl}$}

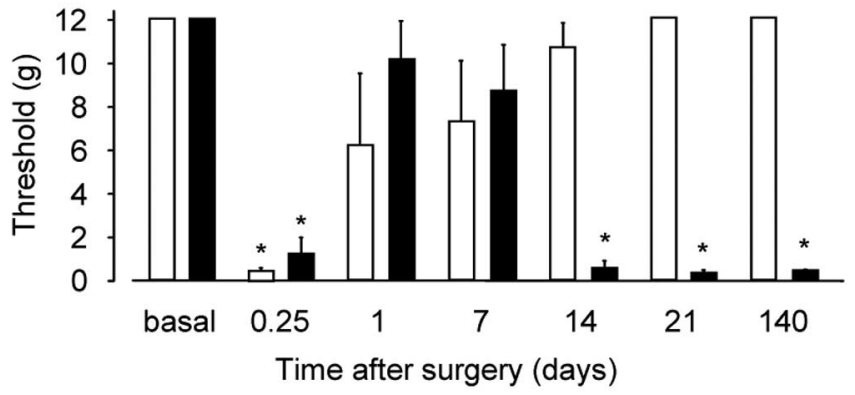

B $\quad \mathrm{SN}-\mathrm{CCl}$

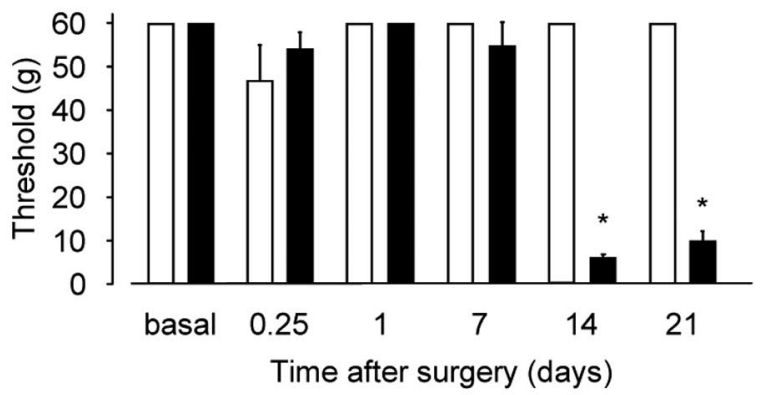

Sham rats $\quad \mathrm{CCl}$ rats

Figure 1. Nociceptive thresholds to mechanical stimulation at various times after unilateral IoN-CCl $(\boldsymbol{A})$ or $\mathrm{SN}-\mathrm{CCl}(\boldsymbol{B})$ surgery in rats. Sensitivity of rats to mechanical stimulations was evaluated with a graded series of von Frey filaments. Each bar is the mean \pm SEM of $n=5-12$ animals. Significant differences were detected from postoperative day 14 and onward in both models when compared with sham counterparts. ${ }^{*} p<0.05$, $\mathrm{Cl}$ rats versus sham animals (Fisher's test).

ment was performed (i.e., IoN-CCI or SN-CCI). For in vitro experiments, PCR results were analyzed by applying a one-way ANOVA with factor dose. For all experiments, in case of significance $(p<0.05)$, the $F$ test was followed by a Fisher's protected least significant difference post hoc test (behavioral and PCR data, minocycline experiments, in vitro experiments) or by a Student's $t$ test (ELISA dosage and PCR after intrathecal injection of rIL-6).

\section{Results}

\section{Establishment of allodynia-like behavior after IoN-CCI} or $\mathrm{SN}-\mathrm{CCI}$

Rats that underwent IoN-CCI surgery exhibited dramatic changes in their responses to mechanical stimuli applied onto the lesioned nerve territory $\left(F_{(1,6)}=25.13 ; p<0.0001\right)$ (Fig. $1 A$ ). After an initial decrease of nociceptive threshold $6 \mathrm{~h}$ after surgery $(1.31 \pm 0.69 \mathrm{~g} ; n=10 ; p<0.001)$, we noted a return to control (basal) values on postoperative days 1 and 7 (10.23 \pm 1.77 and $8.80 \pm 2.07$ g, respectively; $n=10$ in each group; NS compared with basal values). On postoperative day 14 , all IoN-CCI rats presented a marked decrease in nociceptive threshold (0.62 \pm $0.29 \mathrm{~g} ; n=12 ; p<0.001$ ), which was also observed on postoperative days $21(0.39 \pm 0.07 \mathrm{~g} ; n=7 ; p<0.001)$ and $140(0.44 \pm$ $0.04 \mathrm{~g} ; n=5 ; p<0.001)$. As illustrated in Figure $1 A$, sham animals displayed a similar decrease of nociceptive threshold $6 \mathrm{~h}$ after surgery $(0.42 \pm 0.15 \mathrm{~g} ; n=10 ; p<0.001)$, followed by a return to basal values at postoperative days 1 and 7 (6.35 \pm 3.26 and $7.40 \pm 2.82 \mathrm{~g}$, respectively; $n=10$ in each group; NS compared with basal values). From postoperative day 14 onward, sham animals were indistinguishable from their naive counter- parts. The marked decrease of nociceptive threshold occurring $6 \mathrm{~h}$ after surgery in both IoN-CCI and sham rats probably corresponded to a traumatic response, because this allodynia-like behavior gradually disappeared in sham rats, as reported previously by others (Idanpaan-Heikkila and Guilbaud, 1999). The relative hyposensitivity of IoN-CCI rats observed at postoperative days 1 and 7 before the dramatic increase of responsiveness to mechanical stimulation is in line with previous data published on this model (Vos et al., 1994).

In SN-CCI rats, allodynia-like behavior was observed from postoperative day 14 and onward $\left(F_{(1,5)}=39.51 ; p<0.0001\right)$. At this time, $\mathrm{SN}$-CCI rats presented a marked decrease in mechanical threshold compared with sham animals (SN-CCI, $5.58 \pm$ $0.96 \mathrm{~g}$; sham animals, $60 \mathrm{~g} ; p<0.05 ; n=12$ per group), and this change was still observed on postoperative day 21 (Fig. $1 B$ ), in accordance with data in the literature (Attal et al., 1990; Field et al., 1999).

\section{Real time semiquantitative RT-PCR determinations of specific mRNAs}

Whatever the mRNA species considered, tissue levels did not significantly differ between sham and naive rats at any postoperative time tested (data not shown). For the sake of clarity, sham values at every postoperative time tested were pooled into one condition referred to as sham in the following sections. Statistical analyses aimed at comparing IoN-CCI or SN-CCI samples with their respective sham counterparts $(n=4-7)$. The ANOVA values are presented in supplemental Table 1 (available at www. jneurosci.org as supplemental material).

\section{Sensory ganglia}

IoN-CCI rats exhibited a dramatic increase of IL-6 mRNA levels in the TG ipsilateral to the lesion as soon as $6 \mathrm{~h}$ after surgery $(\times 15.40 \pm 3.32, p<0.05$ when compared with respective sham, $n=5)$, and this change persisted until postoperative day 7 ( $\mathrm{d} 1$ : $\times 15.71 \pm 2.76, p<0.01, n=6$; and $\mathrm{d} 7: \times 15.42 \pm 3.00, p<$ $0.001, n=5$ ) (Fig. $2 A$ ). At postoperative days 14 and 21, IL-6 mRNA levels were still elevated, but to a lower extent (d14: $\times 10.74 \pm 1.02, p<0.001, n=6$; and $\mathrm{d} 21: \times 9.21 \pm 1.91, p<$ $0.05, n=4)$. By postoperative day 140 , IL-6 mRNA levels returned to basal values $(\times 1.16 \pm 0.15, n=5)$. In SN-CCI rats, IL-6 mRNA levels in L4-L5 DRG did not differ from basal values $6 \mathrm{~h}$ after the surgery, but an upregulation was observed on postoperative day $1(\times 52.02 \pm 9.90, p<0.001, n=5)$. This change peaked on postoperative day $7(\times 71.60 \pm 8.56, p<0.001, n=5)$ and plateaued from postoperative day 14 until at least day 21 (d14: $\times 51.38 \pm 4.37, p<0.001, n=5$; and $\mathrm{d} 21: \times 49.02 \pm 4.61, p<$ 0.001, $n=5$ ) (Fig. 2A).

As illustrated in Figure $2 B$, ATF3 mRNA levels in TG ipsilateral to the lesion significantly increased $6 \mathrm{~h}$ after IoN-CCI surgery $(\times 3.21 \pm 0.21, p<0.001, n=5)$. This upregulation was further enhanced on postoperative day $1(\times 10.94 \pm 1.80, p<0.001, n=$ $6)$ and then decreased only slightly until postoperative day 21 (d7: $\times 10.10 \pm 0.96, p<0.001, n=5 ; \mathrm{d} 14: \times 7.91 \pm 0.76, p<0.001$, $n=6 ; \mathrm{d} 21: \times 7.90 \pm 1.19, p<0.001, n=4)$. On postoperative day 140, ATF3 mRNA levels returned back to sham values $(\times 1.47 \pm 0.37, n=5)$. In SN-CCI rats, ATF3 mRNA levels in ipsilateral L4-L5 DRG did not significantly change $6 \mathrm{~h}$ after surgery, and exhibited a dramatic upregulation from postoperative day $1-21(\mathrm{~d} 1: \times 16.77 \pm 1.90, p<0.001, n=5$; d7: $\times 18.45 \pm$ $1.83, p<0.001, n=5 ; \mathrm{d} 14: \times 16.33 \pm 0.81, p<0.001, n=5 ; \mathrm{d} 21$ : $\times 15.02 \pm 0.90, p<0.001, n=5)$.

IL-1 $\beta$ mRNA levels in IoN-CCI TG ipsilateral to the lesion did 

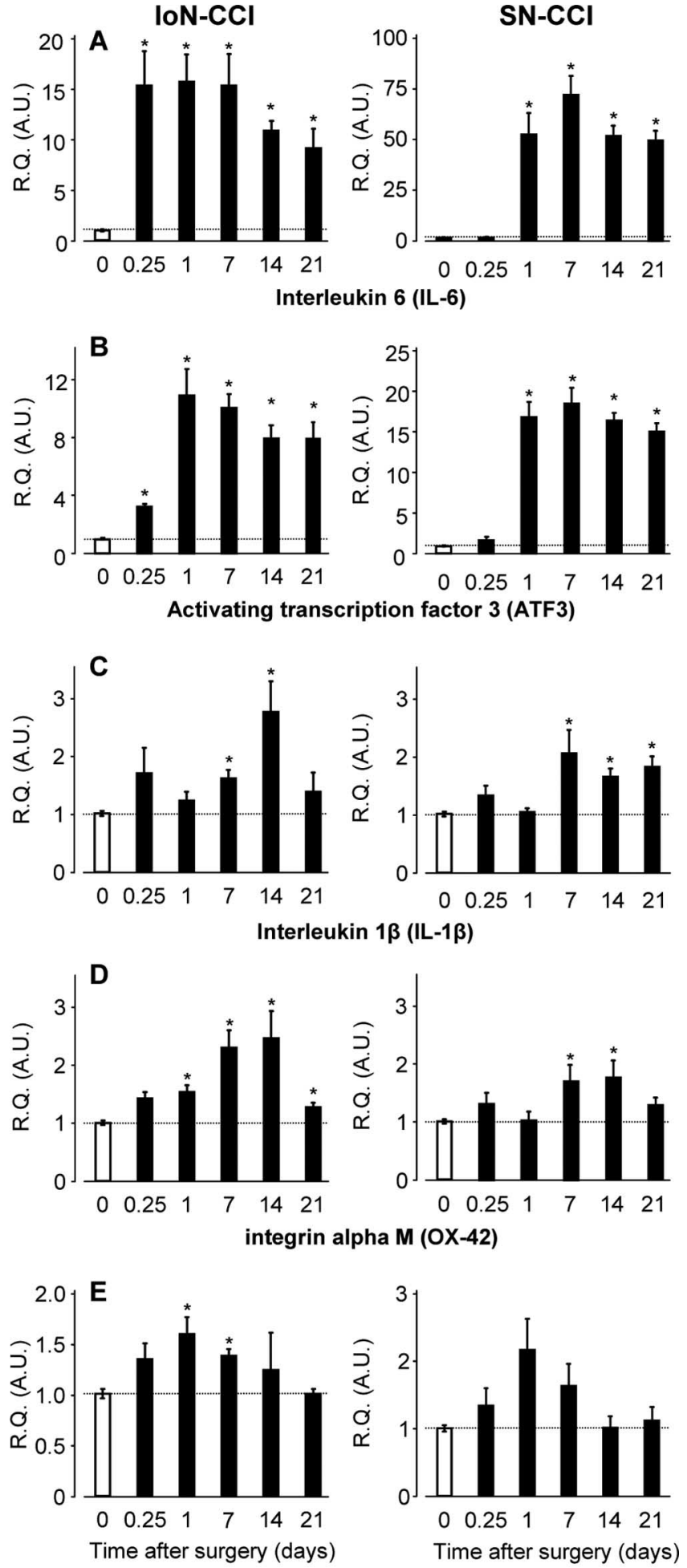

glial fibrillary acidic protein (GFAP)

$\square \quad$ Sham rats

$\mathrm{CCl}$ rats

Figure 2. Time course changes of mRNA levels of IL- $6(\boldsymbol{A}), \operatorname{ATF} 3(\boldsymbol{B}), \mathrm{IL}-1 \beta(\boldsymbol{C}), 0 \mathrm{X}-42(\boldsymbol{D})$, and GFAP $(\boldsymbol{E})$ in ipsilateral trigeminal ganglion and $\mathrm{L} 4-\mathrm{L} 5$ dorsal root ganglia at various times after IoN-CCl or SN-CCl surgery, respectively. R.Q. (A.U.) corresponds to the ratio of specific mRNA over GAPDH mRNA. In each graph, the dotted line represents the R.Q. of respective mRNA determined in sham animals. Each bar is the mean \pm SEM of $n=4-7$ independent determinations. Sham values at every postoperative time are pooled into one condition referred as 0 ; ${ }^{*} p<0.05$, $\mathrm{Cl}$ rats versus sham animals at the same respective postoperative time (Fisher's test). not significantly change before postoperative day 7 when an increase $(\times 1.60 \pm 0.15, p<0.005, n=5)$ was noted (Fig. $2 C)$. This effect was larger on postoperative day $14(\times 2.73 \pm 0.53, p<0.05$, $n=6)$ when allodynia-like behavior occurs. By $21 \mathrm{~d}$, IL- $1 \beta$ mRNA levels were no longer different from sham values $(\times 1.22 \pm 0.29, n=4)$. In SN-CCI rats, IL- $1 \beta$ mRNA levels in L4-L5 DRG increased also on postoperative day 7 , and this effect persisted up to postoperative day $21(\mathrm{~d} 7: \times 2.03 \pm 0.38, p<0.05$, $n=5 ; \mathrm{d} 14: \times 1.63 \pm 0.11, p<0.05, n=5 ; \mathrm{d} 21: \times 1.80 \pm 0.16, p<$ $0.05, n=5)$ (Fig. 2C).

TG mRNA levels of the microglia/macrophage activation marker OX-42 gradually increased from $6 \mathrm{~h}$ after IoN surgery up to maximal values at postoperative days 7 and $14(\mathrm{~d} 7: \times 2.28 \pm$ $0.30, p<0.005, n=5$; and $\mathrm{d} 14: \times 2.31 \pm 0.65, p<0.05, n=6$ ) (Fig. $2 D$ ). On postoperative day 21 , TG OX-42 mRNA levels were still elevated but to a lower extent $(\times 1.27 \pm 0.08, p<0.05, n=$ 4). Finally, at postoperative day $140, \mathrm{OX}-42 \mathrm{mRNA}$ levels were no longer different in IoN-CCI and sham animals. In SN-CCI rats, OX-42 mRNA levels in ipsilateral L4-L5 DRG remained unchanged for the first postoperative day, but were significantly upregulated on postoperative days 7 and $14(\mathrm{~d} 7: \times 1.71 \pm 0.29$, $p<0.05, n=5$; and d14: $\times 1.76 \pm 0.29, p<0.05, n=5)$. At $21 \mathrm{~d}$, L4-L5 DRG OX-42 mRNA levels in SN-CCI rats returned to sham values (Fig. $2 D$ ). OX-42 is a cell adhesion protein found in both microglial cells and macrophages that is overexpressed on activation of either of these cell types (Guillemin and Brew, 2004). As microglial cells are not present within sensory ganglia (Olsson, 1990), the increased OX-42 mRNA levels observed in both TG and DRG probably reflected the recruitment and/or activation of macrophages.

In IoN-CCI rats, GFAP mRNA levels in ipsilateral TG increased significantly on the first day after surgery $(\times 1.58 \pm 0.16$, $p<0.05, n=6)$. This induction was still detectable on postoperative day $7(\times 1.36 \pm 0.07, p<0.01, n=5)$, but not at later postoperative times (Fig. $2 E$ ). In contrast, L4-L5 DRG GFAP mRNA levels tended to increase on in $\mathrm{SN}$-CCI rats postoperative days 1 and 7, but this change did not reach significance (Fig. 2E).

In TG and DRG contralateral to IoN-CCI and SN-CCI, respectively, quantification of the same mRNAs revealed no changes as compared with sham animals, at any time tested (data not shown).

\section{Central tissues}

Unilateral ligation of the infraorbital nerve did not increase IL-6 mRNA (Fig. 3A) and ATF3 mRNA (Fig. 3B) levels in the ipsilateral Sp5c. In contrast, unilateral ligation of the sciatic nerve induced a gradual increase in the levels of both mRNAs in the ipsilateral lumbar dorsal horn starting on postoperative day 1 (IL-6: $\times 2.24 \pm 0.38, p<0.05, n=5$; ATF3: $\times 4.53 \pm$ $0.85, p<0.05, n=5)$ and peaking at postoperative days 7 (IL-6: $\times 4.06 \pm 0.65, p<0.05, n=5$; ATF3: $\times 7.95 \pm 0.91, p<$ $0.001, n=5)$ and $14(\mathrm{IL}-6: \times 3.68 \pm 0.43, p<0.05, n=5$; ATF3: $\times 7.72 \pm 0.84, p<0.001, n=5)$. On postoperative day 21, mRNA levels were still elevated, but to a lower extent (IL-6: $\times 2.14 \pm 0.40, p<0.05, n=5$; ATF3: $5.99 \pm 1.48, p<0.01$, $n=5$ ) (Fig. $3 A, B$ ).

As illustrated in Figure $3 C$, IL- $1 \beta$ mRNA levels in Sp5c ipsilateral to the lesion in IoN-CCI rats were upregulated on postoperative day $1(\times 1.43 \pm 0.10, p<0.05, n=6)$ and reached a peak on postoperative day $7(\times 2.30 \pm 0.50, p<0.05, n=5)$. A significant increase was still observed 1 week later $(\mathrm{d} 14: \times 1.62 \pm 0.24$, $p<0.05, n=6$ ); however, on postoperative day 21 , IL- $1 \beta$ mRNA levels returned to sham values. In the ipsilateral lumbar dorsal 

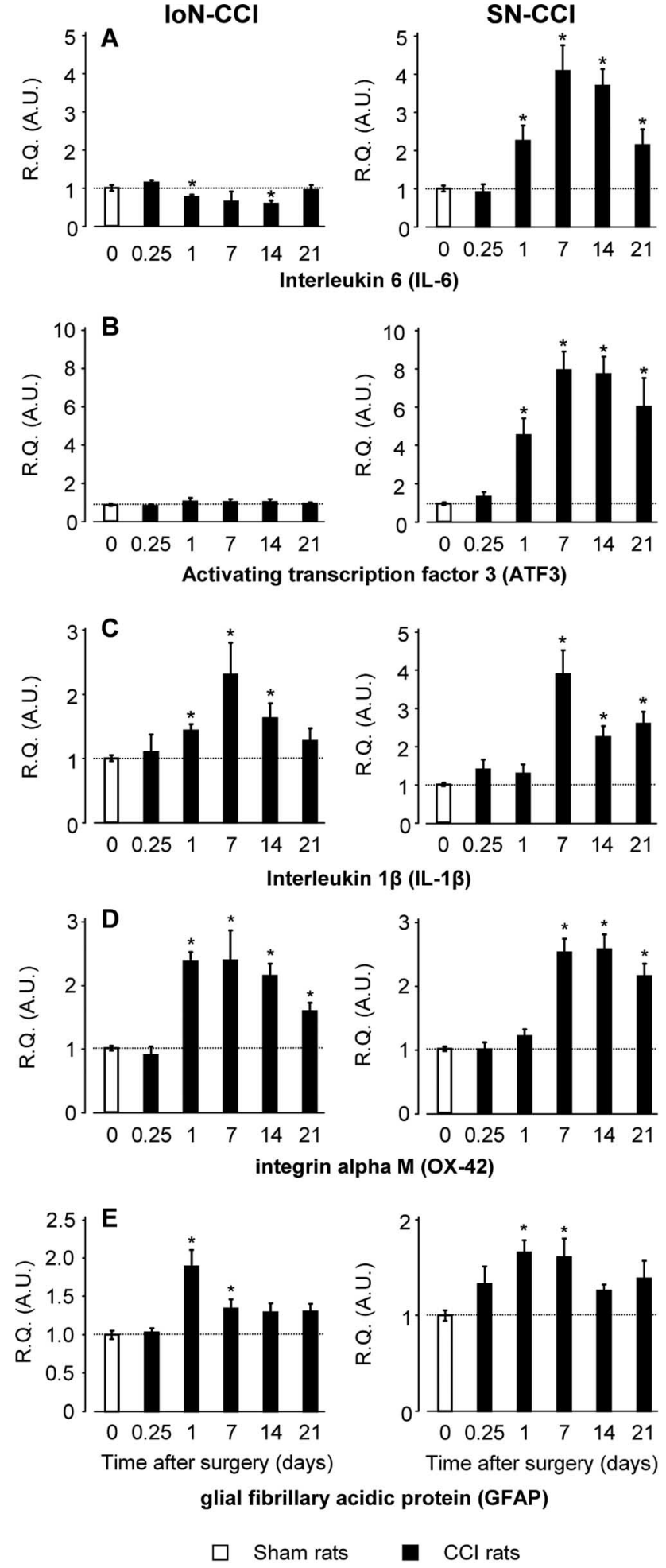

Figure 3. Time course changes in mRNA levels of IL-6 (A), ATF3 (B), IL-1 $\beta(\boldsymbol{C}), 0 \mathrm{OX}-42(\boldsymbol{D})$, and GFAP (E) in the ipsilateral Sp5C and L4 - L5 lumbar dorsal horn at various times after ION-CCl or SN-CCI surgery, respectively. Each bar is the mean \pm SEM of $n=4-7$ independent determinations. Sham values at every postoperative time are pooled into one condition referred as 0 ; ${ }^{*} p<0.05$, $\mathrm{Cl}$ rats versus sham animals at the same respective postoperative time (Fisher's test).

horn of SN-CCI rats, an increase of IL- $1 \beta$ mRNA levels was detected through postoperative days 7-21 (d7: $\times 3.91 \pm 0.59, p<$ 0.001 ; 14 : $\times 2.26 \pm 0.27, p<0.05 ; \mathrm{d} 21: \times 2.59 \pm 0.30, p<0.05$, $n=5$ in each group) (Fig. $3 C$ ).
OX-42 mRNA levels in ipsilateral Sp5c were also markedly increased from postoperative days $1-14$ in IoN-CCI rats (d1: $\times 2.36 \pm 0.14, p<0.001, n=6$; d7: $\times 2.38 \pm 0.46, p<0.05, n=$ 5; d14: $\times 2.13 \pm 0.19, p<0.005, n=6$ ) (Fig. $3 D$ ), with levels lower by postoperative day $21(\times 1.58 \pm 0.12, p<0.005, n=4)$ and normalized by postoperative day $140(\times 1.26 \pm 0.12, n=5)$. In SN-CCI rats, OX-42 mRNA overexpression in ipsilateral lumbar dorsal horn started on postoperative day $7(\times 2.50 \pm 0.21$, $p<0.05, n=5)$ and remained stable until at least postoperative day $21(\mathrm{~d} 14: \times 2.56 \pm 0.22, p<0.001 ; \mathrm{d} 21: \times 2.14 \pm 0.18, p<$ $0.001, n=5$ in each group) (Fig. $3 D$ ).

In both IoN-CCI and SN-CCI rats, GFAP mRNA levels in ipsilateral central tissues exhibited a transient increase through postoperative day 1 (IoN-CCI: $\times 1.89 \pm 0.21, p<0.005, n=6$; SN-CCI: $\times 1.64 \pm 0.12, p<0.05, n=5$ ) to 7 (IoN-CCI: $\times 1.34 \pm$ $0.11, p<0.05, n=5$; SN-CCI: $\times 1.59 \pm 0.19, p<0.05, n=5)$. Determinations at earlier $(6 \mathrm{~h})$ and later $(14$ and $21 \mathrm{~d})$ times after surgery showed no significant differences when compared with sham values (Fig. 3E).

\section{Immunohistological analyses}

In IoN-CCI rats, ATF3 immunostaining was observed in the nuclei of neurons of the TG ipsilateral to the lesion (Fig. $4 A$ ) of the ophthalmic (OPTN) and maxillary (MAXN) areas. In SNCCI rats, a marked increase in ATF3 immunostaining was observed specifically in neurons within L4-L5 DRG ipsilateral to the lesion (Fig. 4C). In addition, ATF3 immunostaining was also enhanced in neurons within the ipsilateral ventral and dorsal horns at the lumbar level (Fig. 4D). In contrast, ATF3 immunostaining in ipsilateral Sp5c did not differ in IoN-CCI rats compared with sham animals (Fig. $4 \mathrm{~B}$ ). Immunostaining of the microglial marker OX-42 revealed the presence of activated microglia in the ipsilateral $\mathrm{Sp} 5 \mathrm{c}$ of IoN-CCI rats and dorsal horn of SN-CCI rats after surgery (Fig. 4, compare $E-G$ with $H$ ), thus confirming the induction of OX-42 evidenced by RT-PCR quantifications.

\section{ELISA measurements of IL-6}

Because IL- 6 is induced in DRG neurons after sciatic nerve lesion (Murphy et al., 1999) and can act in an autocrine manner (Marz et al., 1998; Thier et al., 1999; Faruqi et al., 2001), we sought to determine whether IL-6 protein was released in an autocrine/paracrine or synaptic manner in rats with IoN or SN ligation. Neither sham surgery nor unilateral ligation of the infraorbital nerve increased IL-6 levels in the ipsilateral Sp5c at any time tested (d1: IoN-CCI, $18.8 \pm 5.7 \mathrm{pg} / \mathrm{mg}$ tissue; sham, $16.0 \pm 2.9 \mathrm{pg} / \mathrm{mg}$ tissue; NS, $n=3$ per group; d7: IoN-CCI, $14.5 \pm 3.5 \mathrm{pg} / \mathrm{mg}$ tissue; sham, $14.8 \pm 0.9 \mathrm{pg} / \mathrm{mg}$ tissue; NS, $n=3$ per group; d14: IoN-CCI, $16.6 \pm 3.3 \mathrm{pg} / \mathrm{mg}$ tissue; sham, $17.8 \pm 6.3 \mathrm{pg} / \mathrm{mg}$ tissue; NS, $n=5$ per group). In contrast, IL-6 levels were significantly increased $14 \mathrm{~d}$ after nerve lesion in L4-L5 dorsal horns of SN-CCI rats compared with respective sham tissues $(63.9 \pm 2.8 \mathrm{pg} / \mathrm{mg}$ tissue vs $18 \pm$ $3.8 \mathrm{pg} / \mathrm{mg}$ tissue, respectively; $p<0.05$, Student's $t$ test; $n=5$ per group). Altogether, our data show that IL-6 and ATF3 were differentially altered after injury of the IoN or SN, especially in central tissues.

\section{Effects of minocycline treatment}

Because minocycline has been shown to prevent activation of microglia and associated allodynia-like behavior in several chronic pain models (Raghavendra et al., 2003; Ledeboer et al., 2005; Hains and Waxman, 2006), we next investigated the 
IoN-CCI

TG:OPTN/MAXN area

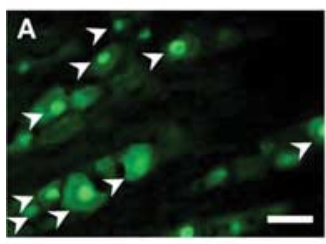

$\mathrm{Sp} 5 \mathrm{c}$

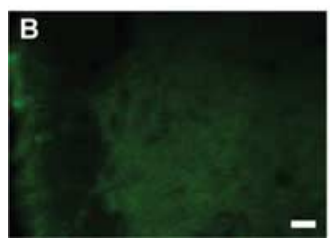

SN-CCI

L4-DRG

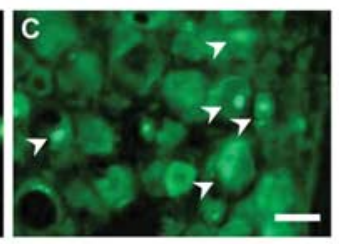

dorsal horn

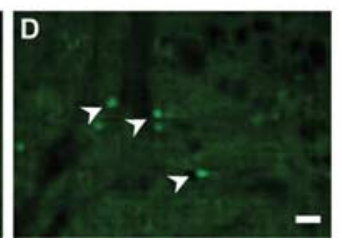

Microglial cells (IoN-CCI)

\section{Postoperative day 1 Postoperative day 7}

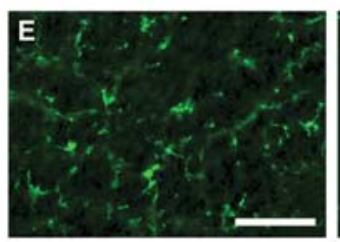

Postoperative day 14

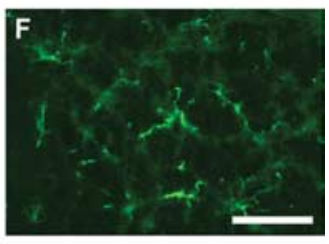

Sham
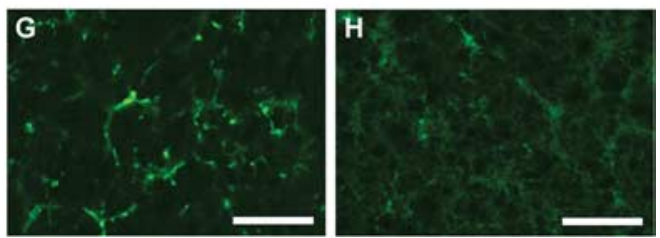

Figure 4. ATF3 and OX-42 immunostaining in sensory ganglia and central tissues in loN-CCI and SN-CCl rats. In loN-CCI rats, an ATF3 immunostaining (in green) was detected (arrowheads) in neurons in the OPTN/MAXN area of the TG ( $\boldsymbol{A}$; note the ATF3-immunoreactive material accumulation in neurons nuclei) but not in the ipsilateral Sp5c $(\boldsymbol{B})$. In contrast, ATF3-labeled neurons were found in both $\mathrm{L} 4-\mathrm{L} 5 \mathrm{DRG}(\boldsymbol{C})$ and dorsal horn $(\boldsymbol{D})$ of SN-CCI rats. OX-42 immunostaining in loN-CCl animals confirmed the presence of activated microglial cells at postoperative days $1(\boldsymbol{E}), 7(\boldsymbol{F})$, and 14 (G) compared with quiescent microglial cells observed in sham rats $(\boldsymbol{H})$. Scale bars, $50 \mu \mathrm{m}$.

potential effects of this drug in both models. Because both IoN-CCI and SN-CCI rats presented significant allodynia-like behavior when compared with sham animals from day 14 after surgery, effects of minocycline on the development of neuropathic pain were assessed at postoperative day 14 . The ANOVA values for RT-PCR experiments are presented in supplemental Table 2 (available at www.jneurosci.org as supplemental material).

Effects of minocycline treatment on allodynia-like behavior in SNCCI and IoN-CCI rats

Before surgery, all groups exhibited similar responses to mechanical stimuli, with no sign of allodynia-like behavior. At the daily dose of $20 \mathrm{mg} / \mathrm{kg}$ intraperitoneally, minocycline was able to partially prevent development of behavioral signs of mechanical allodynia in SN-CCI rats $\left(F_{(1,1)}=6.89 ; p<0.015\right)$ (mechanical threshold values: in SN-CCI rats + vehicle: $8.43 \pm 1.23 \mathrm{~g}, n=7$; in SN-CCI rats + minocycline: $34.86 \pm$ 9.11, $p<0.05, n=7$ ) (Fig. 5). In contrast, the same treatment did not prevent development of allodynia-like responses to mechanical stimuli in IoN-CCI rats [threshold values, $0.71 \pm$ 0.47 and $0.37 \pm 0.04 \mathrm{~g}$ in minocycline- and vehicle-treated rats, respectively; NS, $n$ (vehicle $)=11$ and $n$ (minocycline $)=$ 8]. To determine whether this lack of behavioral effect was related to a different sensitivity to minocycline between the two models, we tested the dose of $40 \mathrm{mg} / \mathrm{kg} / \mathrm{d}$ in IoN-CCI rats. Under this condition, daily administration of minocycline was also unable to prevent the dramatic decrease of nociceptive threshold observed 2 weeks after IoN-CCI $(40 \mathrm{mg} / \mathrm{kg} / \mathrm{d}$ minocycline-treated IoN-CCI rats: $0.59 \pm 0.17 \mathrm{~g}$; vs salinetreated rats: $0.34 \pm 0.06 \mathrm{~g}$; NS, $n=5$ per group).

Effects of minocycline treatment on the levels of mRNA encoding cytokines and neuronal and glial markers in SN-CCI and IoNCCI rats

In sensory ganglia, minocycline, at daily doses of $20 \mathrm{mg} / \mathrm{kg}$ (Fig. $6 A$ ) and $40 \mathrm{mg} / \mathrm{kg}$, significantly reduced (by $\approx 40 \%$ ) IL-6 mRNA overexpression in the ipsilateral TG of IoN-CCI rats (vehicle: $\times 13.92 \pm 1.69, n=9$; Mino20: $\times 7.33 \pm 0.95, n=7$, $p<0.05$; Mino40: $\times 9.10 \pm 1.29, n=5, p<0.05)$. A similar $\approx$
$\mathrm{SN}-\mathrm{CCl}$

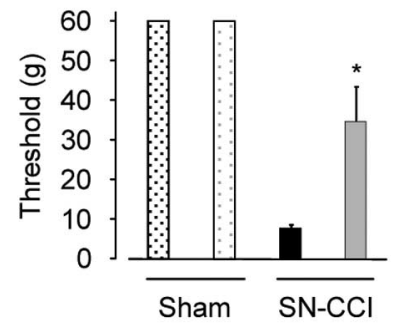

Q Sham rats + vehicle

Sham rats + minocycline $20 \mathrm{mg} / \mathrm{kg} / \mathrm{d}$
IoN-CCI

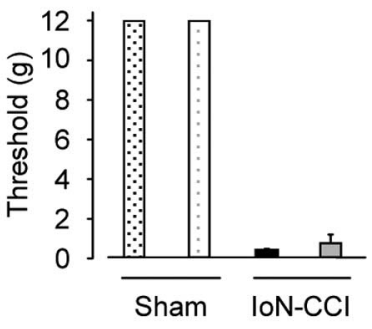

$\mathrm{CCl}$ rats + vehicle

$\mathrm{CCl}$ rats + minocycline $20 \mathrm{mg} / \mathrm{kg} / \mathrm{d}$

Figure 5. Effect of minocycline treatment on mechanical nociceptive threshold of IoN-CCl and SN-CCl rats 2 weeks after the lesion. Minocycline $(20 \mathrm{mg} / \mathrm{kg} / \mathrm{d}$ ) was administered (intraperitoneally) $1 \mathrm{~h}$ before the nerve surgery and then once daily for the following $14 \mathrm{~d}$. Each bar is the mean \pm SEM of $n=5-11$ independent determinations. ${ }^{*} p<0.05$, minocycline-treated rats versus vehicle-treated rats (Fisher's test).

$40 \%$ decrease of IL- 6 mRNA upregulation was observed in ipsilateral L4-L5 DRG of minocycline-treated compared with vehicle-treated SN-CCI rats (vehicle: $\times 53.02 \pm 2.87, n=7$; Mino20: $\times 34.21 \pm 6.29, n=7, p<0.05$ ) (Fig. 6A). In contrast, ATF3 mRNA levels were unaffected by minocycline treatment at either $20 \mathrm{mg} / \mathrm{kg} / \mathrm{d}$ (Fig. $6 B$ ) or $40 \mathrm{mg} / \mathrm{kg} / \mathrm{d}$ in IoN-CCI rats (vehicle: $\times 9.06 \pm 0.82, n=9$; Mino20: $\times 7.51 \pm$ $0.41, n=7$; Mino40: $\times 8.37 \pm 0.55, n=5$ ), whereas they were significantly reduced in L4-L5 DRG of SN-CCI rats (vehicle: $\times 16.73 \pm 0.69, n=7$; Mino20: $\times 10.31 \pm 1.66, n=7, p<$ 0.01 ) (Fig. 6B).

OX-42 mRNA upregulation induced by CCI was significantly reduced by minocycline treatment in IoN-CCI animals (vehicle: $\times 2.19 \pm 0.21, n=9$; Mino20: $\times 1.60 \pm 0.08, n=7$, $p<0.05$ ), whereas in SN-CCI animals, the drug-induced decrease did not reach statistical significance (vehicle: $\times 1.65 \pm$ 0.13, $n=7$; Mino20: $\times 1.35 \pm 0.09, n=7, p=0.08)($ Fig. $6 C$ ).

In contrast, upregulation of mRNA encoding IL- $1 \beta$ was unaffected by minocycline in both TG and DRG of IoN-CCI 
Sensory ganglia
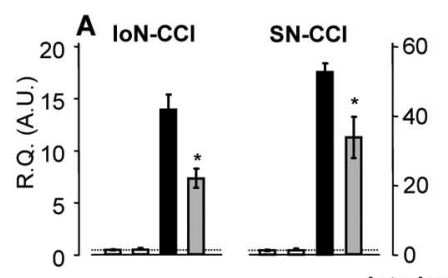

Interleukin 6 (IL-6)
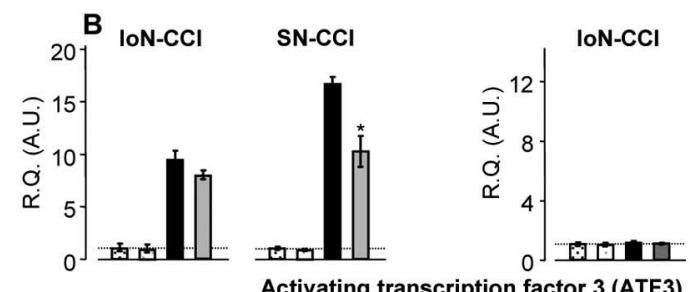

$\mathrm{SN}-\mathrm{CCl}$

Activating transcription factor 3 (ATF3)

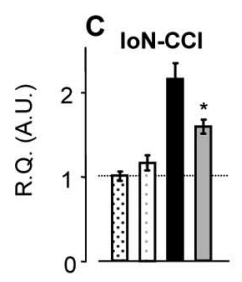

$\mathrm{SN}-\mathrm{CCl}$

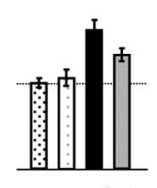

integrin alpha $\mathrm{M}(\mathrm{OX}-42)$

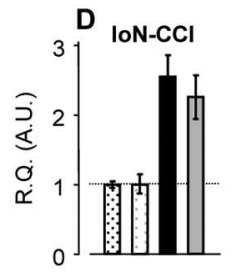

$\mathrm{SN}-\mathrm{CCl}$

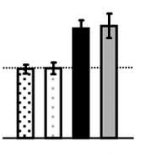

Interleukin $1 \beta(\mathrm{IL}-1 \beta)$

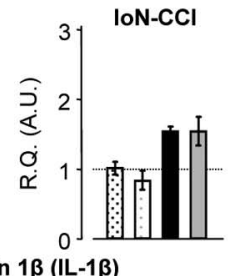

$\mathrm{SN}-\mathrm{CCl}$

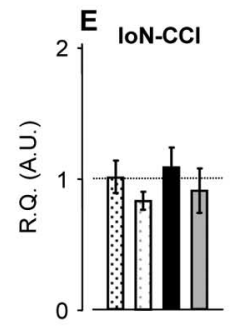

$\mathrm{SN}-\mathrm{CCl}$

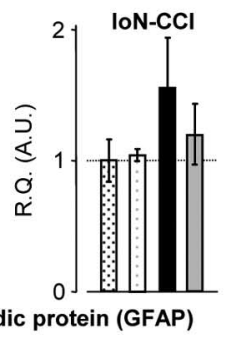

$\mathrm{SN}-\mathrm{CCl}$

Sham rats + vehicle

CCI rats + vehicle

$\square$ Sham rats + minocycline $20 \mathrm{mg} / \mathrm{kg} / \mathrm{d}$

$\square \mathrm{CCl}$ rats + minocycline $20 \mathrm{mg} / \mathrm{kg} / \mathrm{d}$

Figure 6. Effects of minocycline treatment on mRNA levels of IL-6 (A), ATF3 (B), 0X-42 (C), IL-1 $\beta(\boldsymbol{D})$, and GFAP $(\boldsymbol{E})$ in sensory ganglia (TG, L4 - L5 DRG) and central tissues (Sp5C, L4 - L5 lumbar dorsal horn) of IoN-CCl and $\mathrm{SN}-\mathrm{CCl}$ rats detected on postoperative day 14 . Each bar is the mean \pm SEM of $n=5-9$ independent determinations. ${ }^{*} p<0.05$, minocycline-treated rats versus vehicle-treated rats (Fisher's test). R.Q. (A.U.), Ratio of specific mRNA over GAPDH mRNA. In each graph, the dotted line represents the R.Q. of respective mRNA determined in sham + vehicle animals.

and SN-CCI rats, respectively (IoN-CCI: vehicle: $\times 2.53 \pm$ $0.24, n=7$; Mino20: $\times 2.33 \pm 0.33, n=7$; SN-CCI: vehicle: $\times 1.25 \pm 0.12, n=7$; Mino20: $\times 1.60 \pm 0.18, n=7)($ Fig. $6 D)$. Finally, GFAP mRNA levels in both TG and DRG remained unchanged after minocycline treatment in all groups of rats (Fig. 6E).

In $\mathrm{Sp} 5 \mathrm{c}$ and lumbar dorsal horn, minocycline treatment caused a significant reduction of IL- 6 mRNA overexpression in the ipsilateral dorsal horn in SN-CCI rats (vehicle: $\times 3.61 \pm$ 0.42, $n=7$; Mino20: $\times 1.85 \pm 0.33, n=7, p<0.05$ ) (Fig. $6 A$ ) along with a decrease of ATF3 mRNA upregulation (vehicle: $\times 9.67 \pm 1.05, n=7$; Mino20: $4.80 \pm 0.47, n=7, p<0.001$ ) (Fig. $6 \mathrm{~B}$ ). In contrast, this treatment did not affect IL-1 $\beta$ mRNA levels in central tissues in both IoN-CCI and SN-CCI rats (IoN-CCI: vehicle: $\times 1.52 \pm 0.08, n=7$; Mino20: $\times 1.52 \pm$ $0.20, n=7$; SN-CCI: vehicle: $\times 1.99 \pm 0.24, n=7$; Mino20: $\times 2.17 \pm 0.37, n=7$ ) (Fig. 6D).

Surprisingly, minocycline treatment did not reduce OX-42 mRNA upregulation in the Sp5c ipsilateral to the lesion in IoN-CCI rats (vehicle: $\times 2.02 \pm 0.09, n=9$; Mino20: $\times 1.80 \pm$ $0.12, n=7$; Mino40: $\times 1.93 \pm 0.21, n=5$ ), whereas it significantly reduced OX-42 mRNA overexpression in the ipsilateral dorsal horn of SN-CCI rats (vehicle: $\times 2.66 \pm 0.21, n=7$; Mino20: $\times 1.88 \pm 0.10, n=7 ; p<0.05$ ) (Fig. 6C). As noted above for sensory ganglia, GFAP mRNA levels in central tissues were significantly affected by minocycline treatment neither in lesioned nor in sham rats (Fig. 6E).

\section{Implication of IL-6 in the development of allodynia-like behavior after SN-CCI}

The upregulation of IL- 6 in dorsal horn but not $\mathrm{Sp} 5 \mathrm{c}$ after CCI supported the idea that this cytokine could play a role in the development of allodynia-like behavior in SN-CCI rats and consequently be implicated in the differential efficacy of minocycline treatment observed in the two models of neuropathic pain we used in this study. Because IL- 6 is implicated in the recruitment and activation of microglia (Klein et al., 1997), we postulated that this cytokine could participate in the activation of microglia in the dorsal horn of SN-CCI rats. To test this hypothesis, we added IL-6 (1-100 ng/ml) into the culture medium of primary spinal cord microglia. A $6 \mathrm{~h}$ incubation of cell cultures with IL- 6 resulted in a dose-dependent increase of OX-42 mRNA levels with a maximal effect at the dose of $100 \mathrm{ng} / \mathrm{ml}(\times 1.91 \pm 0.13 ; p<0.005$ compared with control condition) (supplemental Fig. 1, available at www. jneurosci.org as supplemental material). Interestingly, cotreatment with minocycline $(100 \mu \mathrm{M})$ did not alter the IL6-evoked induction of OX-42 upregulation (data not shown).

In addition to its ability to enhance OX-42 mRNA expression, we subsequently hypothesized that IL- 6 could also be implicated in ATF3 upregulation in neurons, as it had been reported for other cell types (Cressman et al., 1996). We therefore investigated whether expression of these two genes could be correlated. Indeed, the relative expression of ATF3 mRNA plotted against that of IL-6 mRNA, which is shown in Figure 7, revealed the existence of a positive correlation between ATF3 and IL-6 mRNA upregulations in all tissues assayed (Fig. 7). Because ATF3 is a repressor of IL-6 expression (Gilchrist et al., 2006), we next assessed the ability of IL- 6 to trigger overexpression of ATF3 in primary culture of spinal cord neurons. A $6 \mathrm{~h}$ application of IL-6 $(1-100 \mathrm{ng} / \mathrm{ml})$ to primary coculture of spinal cord neuron/glia elicited a dose-dependent increase of ATF3 mRNA specifically in neurons $\left(F_{(4,14)}=18.47 ; p<\right.$ 0.0001 ) (Fig. $8 A$ ). Interestingly, this effect was detected as soon as $1 \mathrm{~h}$ after IL- 6 addition to the culture medium. As expected from a direct action of IL- 6 on neurons, the cytokine was also found to induce a dose-dependent upregulation of ATF3 in cultures enriched in spinal cord neurons $\left(F_{(4,11)}=\right.$ 37.60; $p<0.0001$ ) (Fig. $8 A$ ), and this effect persisted in the presence of minocycline (100 $\mu \mathrm{M}$; data not shown). 

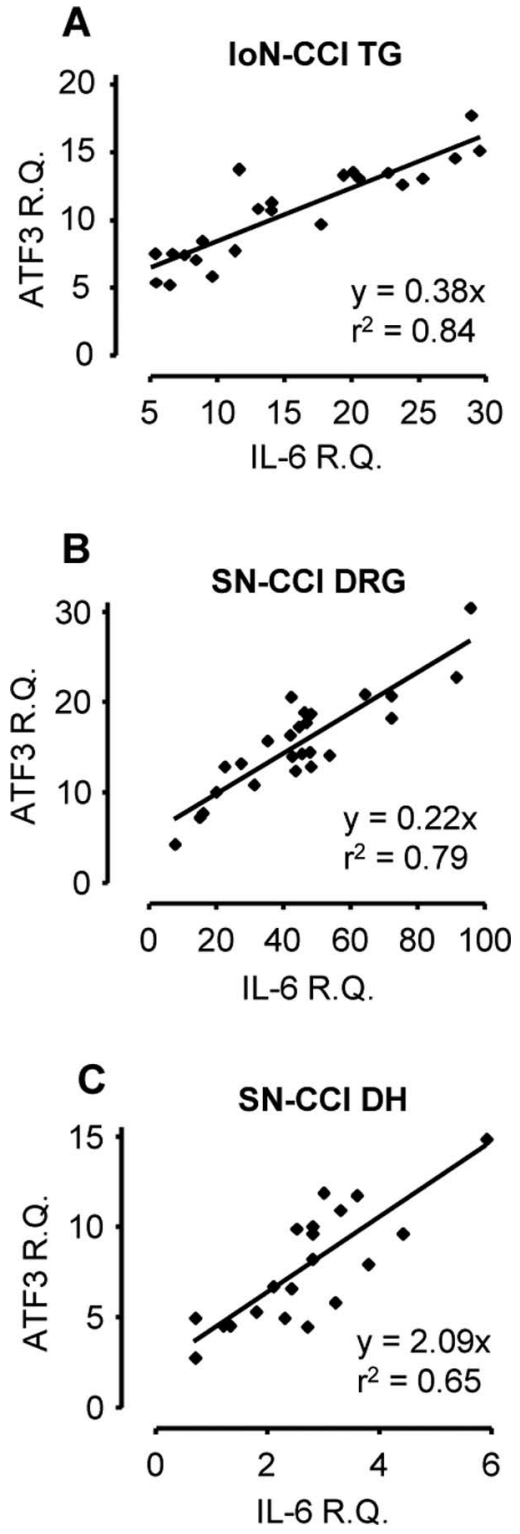

Figure 7. Relative expression of ATF3 mRNA (ordinate) plotted against that of IL-6 mRNA (abscissa) shows a close association between upregulation of these two genes after IoN-CCl and $\mathrm{SN}-\mathrm{CCl}$. Each point corresponds to one rat. Regression analysis revealed a significant correlation between ATF3 and IL-6 mRNA levels in trigeminal ganglion of IoN-CCI rats $(\boldsymbol{A})$, in L4 -L5 DRG of $\mathrm{SN}-\mathrm{CCl}$ rats $(\boldsymbol{B})$, and in the lumbar dorsal horn of $\mathrm{SN}-\mathrm{CCl}$ rats (C). R.Q. (A.U.), Ratio of specific mRNA over GAPDH mRNA.

Finally, we tested whether IL-6 ( $1 \mu \mathrm{g} / 25 \mu \mathrm{l})$ administered intrathecally could increase ATF3 and OX-42 mRNA expression within the dorsal horn of naive rats. As illustrated in Figure $8 B$, a marked upregulation of both ATF3 and OX-42 mRNA levels was observed in the L4-L5 dorsal horns $6 \mathrm{~h}$ after this treatment $(\times 5.03 \pm 0.56, p<0.005 ; \times 2.98 \pm 0.20, p<$ 0.001 , respectively; $n=4)$ compared with intrathecal injection of the vehicle $(n=4)$. These data thus confirm the ability of IL- 6 to trigger ATF3 and OX-42 induction in vivo (Fig. $8 B$ ). However, no allodynia-like responses to mechanical stimulations were detected 1.5 and $3 \mathrm{~h}$ after IL- 6 intrathecal injection (data not shown), indicating that allodynia-like behavior observed after SN-CCI is probably the consequence of long-term plasticity occurring in the dorsal horn.

\section{Discussion}

Our study describes major differences in signaling pathways implicated in the development of abnormal pain states after infraorbital or sciatic nerve injury and provides evidence that interactions between injured neurons and glial cells play key roles in underlying mechanisms. In line with this conclusion, subchronic treatment with minocycline partially prevents the development of allodynia-like behavior in SN-CCI rats but not in IoN-CCI rats, possibly through a direct inhibitory influence on IL- 6 production by spinal neurons.

\section{Cytokines, neuronal, and glial markers in sensory ganglia}

After sciatic nerve damage, the different genes we assessed exhibited an upregulation in the L4-L5 DRG of SN-CCI rats. These results confirm and extend previous data on this model (Chudler et al., 1997; Tsujino et al., 2000; Sweitzer et al., 2001; Obata et al., 2003; Lee et al., 2004). We also report a similar induction of these genes in the TG of IoN-CCI rats. However, overexpression of IL-6 and OX-42 occurred earlier in IoNCCI compared with SN-CCI animals. This difference in respective response onset is opposite to that expected from an inflammatory reaction that would have been higher because of the four chromic catgut ligations in SN-CCI (Bennett and Xie, 1988 ) compared with only two ligations in IoN-CCI (Vos et al., 1994). Indeed, the difference between the effects of ligation on the two nerves is more probably related to the presence of a higher density of myelinated fibers in the IoN (50\%) than in the SN (20\%) (Young, 1977). Guilbaud et al. (1993) clearly showed that myelinated fibers are the most vulnerable to CCI. In additional support of this interpretation, ATF3, which is considered as a marker of neuronal response to injury (Hai and Hartman, 2001) and is essentially expressed by medium to large neurons (Obata et al., 2003; Tsuzuki et al., 2003) from which originate myelinated fibers (Lazarov, 2002), was also found to exhibit a more robust and faster upregulation after IoN-CCI compared with SN-CCI. Interestingly, IL-6 mRNA increase in TG of IoN-CCI rats was detected as soon as $1.5 \mathrm{~h}$ after surgery with mRNA levels $(\times 33.85 \pm 7.75)$ even higher than at later postoperative times (Fig. $2 \mathrm{~A}$ ). In contrast, SNCCI-induced upregulation of IL- 6 mRNA in DRG peaked only 1 week after surgery. Such a rapid induction of IL- 6 mRNA levels in TG of IoN-CCI rats could suggest an activitydependent induction (Sallmann et al., 2000), in opposition to the slower induction observed in the DRGs of SN-CCI rats, which would require retrograde signals (Murphy et al., 1999). Altogether, these data suggest a different response of sensory ganglia cells to injury in IoN-CCI versus SN-CCI rats.

Minocycline treatment also revealed differences in signaling pathways activated after IoN-CCI or SN-CCI. Minocycline has been shown to exert neuroprotective effects by either reducing microglial activation (Yrjanheikki et al., 1999) or acting directly on neurons (Lin et al., 2001; Alano et al., 2006; González et al., 2007). Induction of ATF3 is triggered by signals that also induce cellular damage (Chen et al., 1996). Because ATF3 is able to repress the activity of its own promoter (Hai and Hartman, 2001), the maintained upregulation detected in sensory neurons after CCI (Fig. $2 B$ ) represents a reliable marker of ongoing neuronal damage (Hai and Hartman, 2001). Interestingly, preemptive minocycline treatment reduced ATF3 upregulation detected $14 \mathrm{~d}$ after SN-CCI, thus suggesting a partial protection of the corresponding neurons. In contrast, this treatment did not affect ATF3 upregulation in IoN-CCI rats, indicating the persistence of neuronal damage 
A
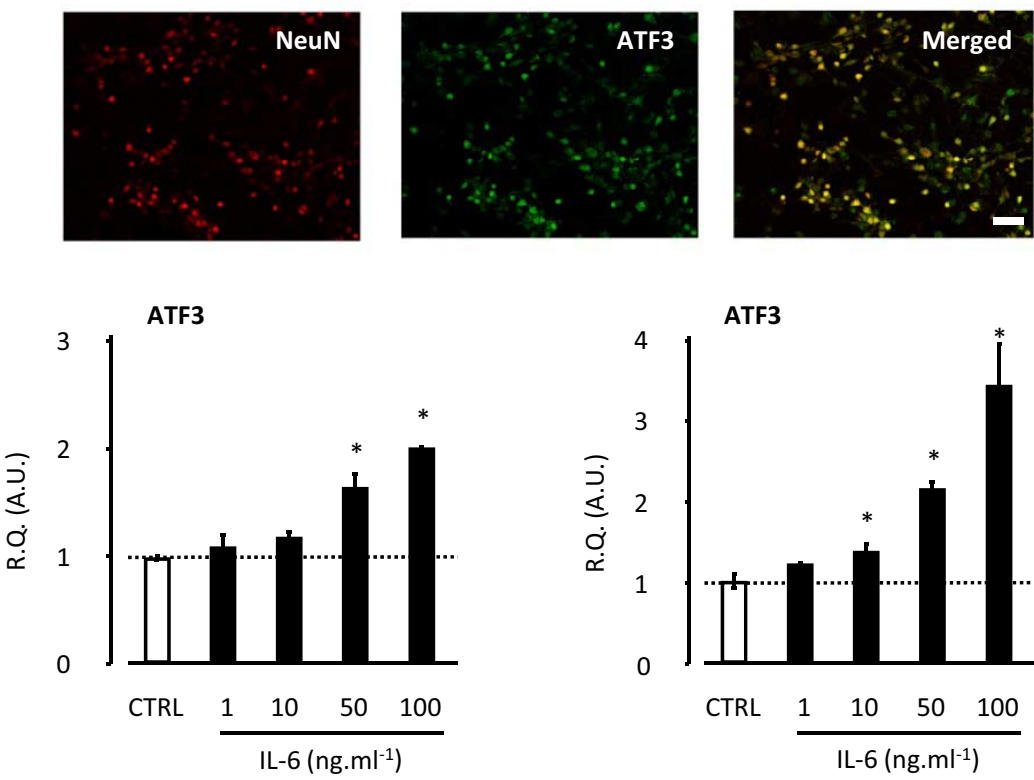

Mixed culture spinal cord neurons / glia

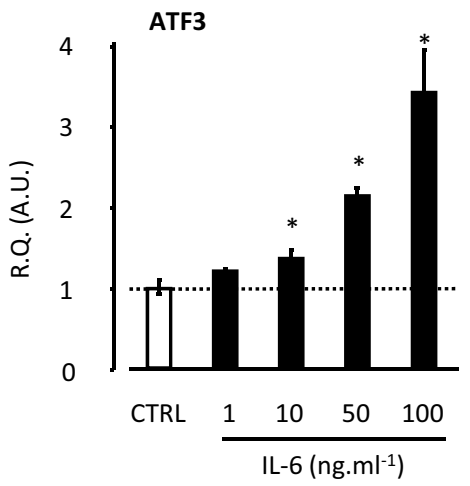

Enriched spinal cord neurons culture

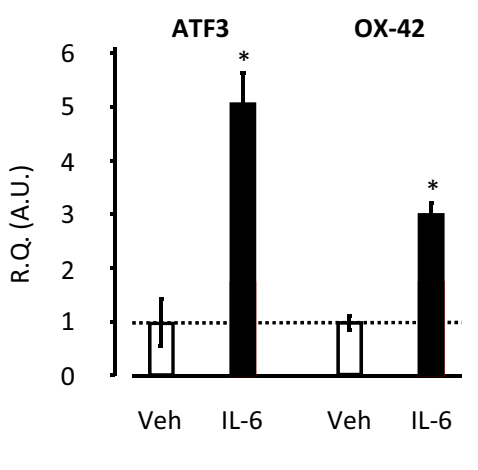

Intrathecal injection of rIL-6 (1 ng.25 $\left.\mu^{-1}\right)$

Figure 8. IL-6-induced overexpression of ATF3 mRNA in spinal cord neurons. A, Addition of IL-6 (1-100 ng) to the medium of primary cultures of mixed spinal glia-neurons or of enriched spinal neurons produced a dose-dependent increase in neuronal expression of ATF3 mRNA. Each bar is the mean \pm SEM of $n=3$ independent determinations. ${ }^{*} p<0.05$ compared with control condition (Fisher's test). In primary cultures of mixed spinal glia-neurons incubated with IL-6 (100 ng), ATF3 immunostaining colocalized with the neuronal marker NeuN, indicating neuronal expression of ATF3. No ATF3 immunostaining was detected in the absence of IL-6. Scale bar, $50 \mu \mathrm{m}$. B, Intrathecal injection of rlL-6 (1 $\mu \mathrm{g} / 25 \mu \mathrm{l})$ in naive rats induced an upregulation of ATF3 and 0X-42 mRNA in L4 -L5 dorsal horn $6 \mathrm{~h}$ after injection. Each bar is the mean \pm SEM of $n=4$ independent determinations. * $p<0.05$ compared with control condition (Student's $t$ test). R.Q. (A.U.), Ratio of specific mRNA over GAPDH mRNA. In $\boldsymbol{A}$, the dotted line represents the R.Q. of ATF3 mRNA determined in control conditions. In $\boldsymbol{B}$, the dotted line represents the R.Q. of respective mRNA determined in vehicle-treated animals.

in treated animals. In conclusion, our results indicate that, in the CCI models we used, neurons of the sensory ganglia are the first cells to respond to nerve injury. However, the signaling pathways involved appear to be different in case of lesion to the IoN versus the SN. In particular, additional studies are needed to determine, on one hand, the factors responsible for IL- 6 induction in the trigeminal versus the dorsal root ganglia, and, on the other hand, those factors responsible for the maintenance of ATF3 overexpression in minocycline-treated IoNCCI rats.

\section{Cytokines, neuronal, and glial markers in central structures}

As described previously after nerve injury (Hains et al., 2004; Verge et al., 2004), we observed microglia activation in both SN-CCI and IoN-CCI rats with a concomitant upregulation of IL- $1 \beta$ mRNA levels, supporting the idea that IL- $1 \beta$ is produced by ameboid microglia (Giulian et al., 1986; Ferrari et al., 1997). Activated microglia is a key feature of pain hypersensitivity, and recent data have shown that its blockade prevents development of allodynia-like behavior in animal models of neuropathic pain (Raghavendra et al., 2003; Ledeboer et al., 2005; Piao et al., 2006). However, it is not clear whether this blockade is caused by intrinsic effects of the drug on microglia or by an inhibitory action upstream on signaling mechanisms at the origin of microglia activation. Microglia activation can be triggered by numerous stimuli such as glutamate (Tikka and Koistinaho, 2001), ATP (Ferrari et al., 1997), or nitric oxide (Roy et al., 2006), which are released by injured neurons (Minc-Golomb et al., 1996). In addition, IL-6-deficient mice exhibit altered microglia activation (Klein et al., 1997; Galiano et al., 2001) and neuronal overexpression of IL-6 potentiates glia activation (Fattori et al., 1995), further suggesting a role for IL-6 in microglial activation. We confirm here that IL-6 can directly promote upregulation of OX-42 mRNA in spinal cord microglial cells. Arruda et al. (1998) reported that neurons of the dorsal horn could produce IL- 6 after nerve damage. Our data also support this observation, because expression of both IL-6 and ATF3 appeared positively correlated and preceded microglia activation. However, because IL- 6 can also be expressed by microglial cells (Milligan et al., 2000; Ledeboer et al., 2005), additional experiments would be required to determine the precise cell type producing and releasing IL-6 after SN-CCI. Altogether, we propose that, after SN-CCI, IL-6 might trigger microglia activation. Accordingly, the partial reduction of microglia activation in SN-CCI rats under minocycline treatment could be explained by a drug-induced inhibition of IL- 6 production rather than reflecting a direct effect on microglial cells. Indeed, IL-6-induced microglia activation is not mediated through a minocycline-sensitive pathway, because coapplication of IL- 6 and minocycline in vitro did not alter IL-6-induced OX-42 upregulation. Furthermore, minocycline was found to reduce IL- 6 and ATF3 inductions already on the first postoperative day, at a time when no microglia activation has yet occurred in dorsal horn of SN-CCI rats (supplemental Fig. 2, available at www.jneurosci.org as supplemental material). Finally, we demonstrate that IL-6 can induce ATF3 upregulation by a direct action on neurons. Hence, it can be proposed that IL-6 upregulation could be responsible for subsequent ATF3 upregulation and downstream cascade of transcription events in the dorsal horn of 

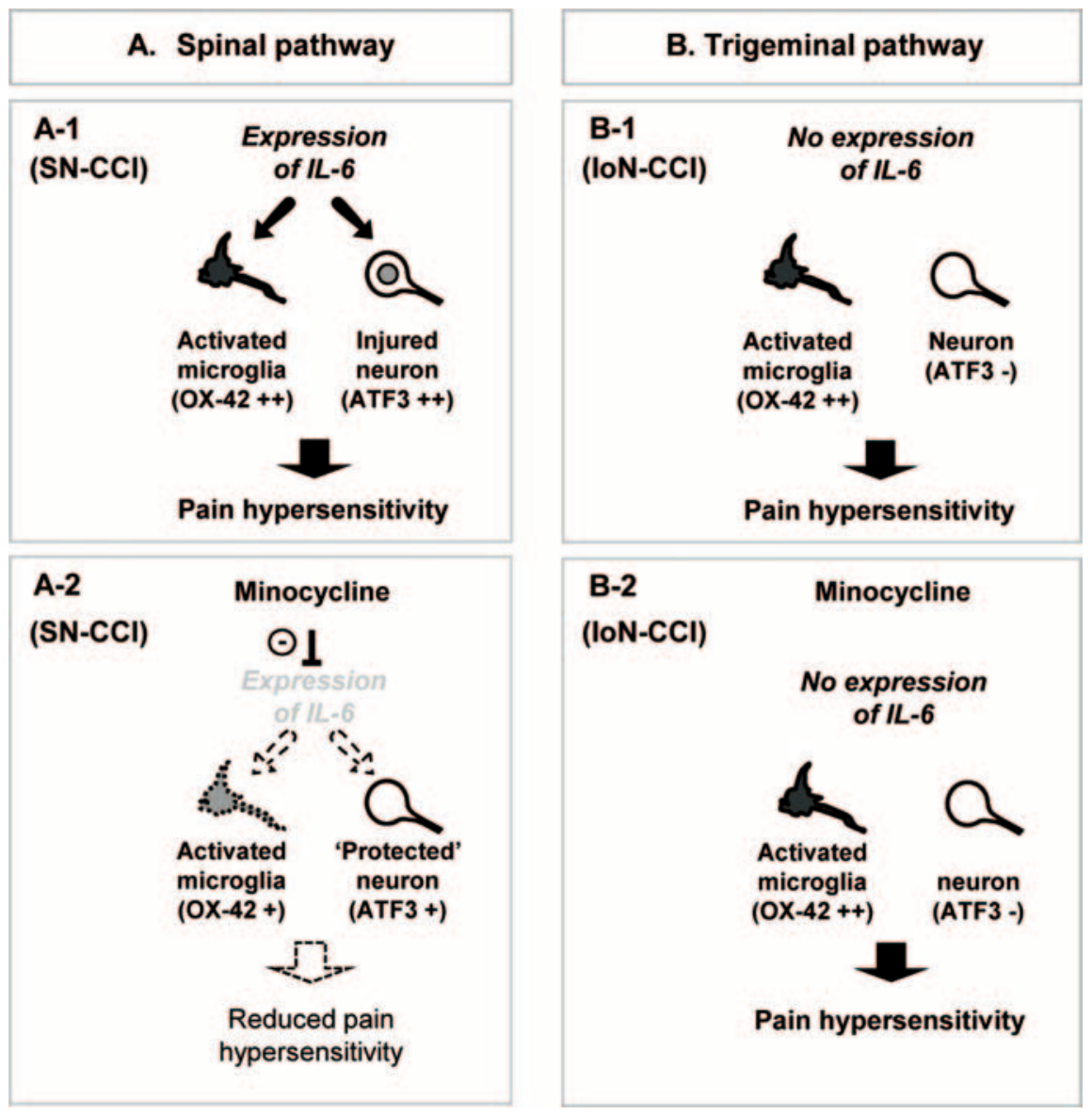

Figure 9. Schematic representation of the possible differential signaling pathways mediating pain hypersensitivity after SN-CCl or loN-CCI. A-1, In SN-CCI rats, IL-6 could play a key role in the induction of ATF3 and subsequent cascade of transcription events as well as in microglia activation. $\boldsymbol{B}-\mathbf{1}$, In contrast, in loN-CCl rats, Sp5c neurons exhibited neither ATF3 nor IL-6 overexpression, suggesting that microglial activation is mediated by IL-6-independent pathways. $A-2$, Minocycline treatment inhibited CCl-induced IL-6 expression, which subsequently caused a decrease in 0X-42 and ATF3 upregulations. B-2, In contrast, IL-6independent microglial activation observed in IoN-CCI rats was not affected by minocycline, thus explaining why the drug did not reduce pain hypersensitivity in these animals.

SN-CCI rats. Altogether, our data strongly suggest that, after $\mathrm{SN}-\mathrm{CCI}$, IL-6 is one of the major protagonists at the origin of the pathophysiological mechanisms underlying pain hypersensitivity (Fig. 9).

In contrast, in IoN-CCI rats, $\mathrm{Sp} 5 \mathrm{c}$ neurons exhibited neither ATF3 nor IL-6 overexpression, and minocycline treatment was unable to prevent microglia activation. These data are coherent with the idea that minocycline targets IL-6 production pathways, thus explaining its lack of effect in IoN-CCI rats (Fig. 9). However, some discrepancy has to be discussed between our data and those of Piao et al. (2006) who described effective prevention by minocycline of allodynia-like behavior and microglia activation in rats with transection of both inferior alveolar and mental nerves at the cephalic level. The latter model differs markedly from our IoN-CCI cephalic model (loose ligatures of the fibers innervating the vibrissae territory vs transection of fibers innervating adjacent territories in the model of Piao et al.). In particular, previous studies showed that, after cutting a nerve, most of degenerating cells are smalldiameter DRG neurons at the origin of unmyelinated fibers (Janig and McLachlan, 1984), whereas the vast majority of degenerating neurons after CCI are large-diameter cells at the origin of myelinated fibers (Guilbaud et al., 1993). Conse- quently, lesion-induced signals emitted by primary afferent fibers very probably differ in the model of Piao et al. (2006) and the IoN-CCI model used here. Because minocycline exerts a stimulusdependent action (Nikodemova et al., 2006), it is probable that microglia activation observed in rats with transected nerves is mediated by minocyclinesensitive pathways, whereas, under our conditions, IoN-CCI-induced microglia activation is minocycline insensitive.

In conclusion, our data support the idea that distinct pathophysiological mechanisms contribute to the development of cephalic versus extracephalic neuropathic pain. A major difference is the overexpression of IL-6 detected in the lumbar dorsal horn of SN-CCI rats but not in the Sp5c of IoN-CCI rats. Our finding that IL-6 can elicit microglia activation and ATF3 overexpression both in vitro and in vivo suggests an essential role played by this cytokine in the alterations occurring in the dorsal horn of $\mathrm{SN}-\mathrm{CCI}$ rats. Consequently, the minocycline-induced decrease in spinal IL-6 production likely plays an important role in its efficacy to prevent pain hypersensitivity development. Determination of the factors responsible for microglia activation in the $\mathrm{Sp} 5 \mathrm{c}$ of IoN-CCI rats should be of great interest to develop new treatments against cephalic neuropathic pain symptoms.

\section{References}

Alano CC, Kauppinen TM, Valls AV, Swanson RA (2006) Minocycline inhibits poly(ADPribose) polymerase-1 at nanomolar concentrations. Proc Natl Acad Sci USA 103:9685-9690.

Arruda JL, Colburn RW, Rickman AJ, Rutkowski MD, DeLeo JA (1998) Increase of interleukin- 6 mRNA in the spinal cord following peripheral nerve injury in the rat: potential role of IL- 6 in neuropathic pain. Brain Res Mol Brain Res 62:228-235.

Attal N, Jazat F, Kayser V, Guilbaud G (1990) Further evidence for "painrelated" behaviours in a model of unilateral peripheral mononeuropathy. Pain 41:235-251.

Bennett GJ (2004) Neuropathic pain in the orofacial region: clinical and research challenges. J Orofac Pain 18:281-286.

Bennett GJ, Xie YK (1988) A peripheral mononeuropathy in rat that produces disorders of pain sensation like those seen in man. Pain 33:87-107.

Benoliel R, Eliav E, Tal M (2001) No sympathetic nerve sprouting in rat trigeminal ganglion following painful and non-painful infraorbital nerve neuropathy. Neurosci Lett 297:151-154.

Chen BP, Wolfgang CD, Hai T (1996) Analysis of ATF3, a transcription factor induced by physiological stresses and modulated by gadd153/ Chop10. Mol Cell Biol 16:1157-1168.

Chudler EH, Anderson LC, Byers MR (1997) Trigeminal ganglion neuronal activity and glial fibrillary acidic protein immunoreactivity after inferior alveolar nerve crush in the adult rat. Pain 73:141-149.

Cressman DE, Greenbaum LE, DeAngelis RA, Ciliberto G, Furth EE, Poli V, Taub R (1996) Liver failure and defective hepatocyte regeneration in interleukin-6-deficient mice. Science 274:1379-1383.

Faruqi TR, Gomez D, Bustelo XR, Bar-Sagi D, Reich NC (2001) Rac1 medi- 
ates STAT3 activation by autocrine IL-6. Proc Natl Acad Sci USA 98:9014-9019.

Fattori E, Lazzaro D, Musiani P, Modesti A, Alonzi T, Ciliberto G (1995) IL-6 expression in neurons of transgenic mice causes reactive astrocytosis and increase in ramified microglial cells but no neuronal damage. Eur J Neurosci 7:2441-2449.

Ferrari D, Chiozzi P, Falzoni S, Hanau S, Di Virgilio F (1997) Purinergic modulation of interleukin-1 beta release from microglial cells stimulated with bacterial endotoxin. J Exp Med 185:579-582.

Field MJ, Bramwell S, Hughes J, Singh L (1999) Detection of static and dynamic components of mechanical allodynia in rat models of neuropathic pain: are they signalled by distinct primary sensory neurones? Pain 83:303-311.

Galiano M, Liu ZQ, Kalla R, Bohatschek M, Koppius A, Gschwendtner A, Xu S, Werner A, Kloss CU, Jones LL, Bluethmann H, Raivich G (2001) Interleukin-6 (IL6) and cellular response to facial nerve injury: effects on lymphocyte recruitment, early microglial activation and axonal outgrowth in IL6-deficient mice. Eur J Neurosci 14:327-341.

Gilchrist M, Thorsson V, Li B, Rust AG, Korb M, Roach JC, Kennedy K, Hai T, Bolouri H, Aderem A (2006) Systems biology approaches identify ATF3 as a negative regulator of Toll-like receptor 4. Nature 441:173-178.

Giulian D, Baker TJ, Shih LC, Lachman LB (1986) Interleukin 1 of the central nervous system is produced by ameboid microglia. J Exp Med 164:594-604.

González JC, Egea J, Del Carmen Godino M, Fernandez-Gomez FJ, SánchezPrieto J, Gandía L, García AG, Jordán J, Hernández-Guijo JM (2007) Neuroprotectant minocycline depresses glutamatergic neurotransmission and $\mathrm{Ca}^{2+}$ signalling in hippocampal neurons. Eur J Neurosci 26:2481-2495.

Goslin K, Asmussen H, Banker G (1998) Rat hippocampal neurons in lowdensity culture. In: Culturing nerve cells (Banker G, Goslin K, eds), pp 339-370. Cambridge, MA: MIT.

Guilbaud G, Gautron M, Jazat F, Ratinahirana H, Hassig R, Hauw JJ (1993) Time course of degeneration and regeneration of myelinated nerve fibres following chronic loose ligatures of the rat sciatic nerve: can nerve lesions be linked to the abnormal pain-related behaviours? Pain 53:147-158.

Guillemin GJ, Brew BJ (2004) Microglia, macrophages, perivascular macrophages, and pericytes: a review of function and identification. J Leukoc Biol 75:388-397.

Hai T, Hartman MG (2001) The molecular biology and nomenclature of the activating transcription factor/cAMP responsive element binding family of transcription factors: activating transcription factor proteins and homeostasis. Gene 273:1-11.

Hains BC, Waxman SG (2006) Activated microglia contribute to the maintenance of chronic pain after spinal cord injury. J Neurosci 26:4308-4317.

Hains BC, Saab CY, Klein JP, Craner MJ, Waxman SG (2004) Altered sodium channel expression in second-order spinal sensory neurons contributes to pain after peripheral nerve injury. J Neurosci 24:4832-4839.

Idänpään-Heikkilä JJ, Guilbaud G (1999) Pharmacological studies on a rat model of trigeminal neuropathic pain: baclofen, but not carbamazepine, morphine or tricyclic antidepressants, attenuates the allodynia-like behaviour. Pain 79:281-290.

Jänig W, McLachlan E (1984) On the fate of sympathetic and sensory neurons projecting into a neuroma of the superficial peroneal nerve in the cat. J Comp Neurol 225:302-311.

Kayser V, Aubel B, Hamon M, Bourgoin S (2002) The antimigraine 5-HT $1 \mathrm{~B} / 1 \mathrm{D}$ receptor agonists, sumatriptan, zolmitriptan and dihydroergotamine, attenuate pain-related behaviour in a rat model of trigeminal neuropathic pain. Br J Pharmacol 137:1287-1297.

Klein MA, Möller JC, Jones LL, Bluethmann H, Kreutzberg GW, Raivich G (1997) Impaired neuroglial activation in interleukin-6 deficient mice. Glia 19:227-233.

Krady JK, Basu A, Levison SW, Milner RJ (2002) Differential expression of protein tyrosine kinase genes during microglial activation. Glia 40:11-24.

Lazarov NE (2002) Comparative analysis of the chemical neuroanatomy of the mammalian trigeminal ganglion and mesencephalic trigeminal nucleus. Prog Neurobiol 66:19-59.

Ledeboer A, Sloane EM, Milligan ED, Frank MG, Mahony JH, Maier SF,
Watkins LR (2005) Minocycline attenuates mechanical allodynia and proinflammatory cytokine expression in rat models of pain facilitation. Pain 115:71-83.

Lee HL, Lee KM, Son SJ, Hwang SH, Cho HJ (2004) Temporal expression of cytokines and their receptors mRNAs in a neuropathic pain model. Neuroreport 15:2807-2811.

Lin S, Zhang Y, Dodel R, Farlow MR, Paul SM, Du Y (2001) Minocycline blocks nitric oxide-induced neurotoxicity by inhibition p38 MAP kinase in rat cerebellar granule neurons. Neurosci Lett 315:61-64.

Livak KJ, Schmittgen TD (2001) Analysis of relative gene expression data using real-time quantitative PCR and the 2(-Delta Delta C(T)) Method. Methods 25:402-408.

März P, Cheng JG, Gadient RA, Patterson PH, Stoyan T, Otten U, Rose-John S (1998) Sympathetic neurons can produce and respond to interleukin 6. Proc Natl Acad Sci USA 95:3251-3256.

Milligan ED, Mehmert KK, Hinde JL, Harvey LO, Martin D, Tracey KJ, Maier SF, Watkins LR (2000) Thermal hyperalgesia and mechanical allodynia produced by intrathecal administration of the human immunodeficiency virus-1 (HIV-1) envelope glycoprotein, gp120. Brain Res 861:105-116.

Minc-Golomb D, Yadid G, Tsarfaty I, Resau JH, Schwartz JP (1996) In vivo expression of inducible nitric oxide synthase in cerebellar neurons. J Neurochem 66:1504-1509.

Murphy PG, Grondin J, Altares M, Richardson PM (1995) Induction of interleukin-6 in axotomized sensory neurons. J Neurosci 15:5130-5138.

Murphy PG, Ramer MS, Borthwick L, Gauldie J, Richardson PM, Bisby MA (1999) Endogenous interleukin-6 contributes to hypersensitivity to cutaneous stimuli and changes in neuropeptides associated with chronic nerve constriction in mice. Eur J Neurosci 11:2243-2253.

Nikodemova M, Duncan ID, Watters JJ (2006) Minocycline exerts inhibitory effects on multiple mitogen-activated protein kinases and IkappaBalpha degradation in a stimulus-specific manner in microglia. J Neurochem 96:314-323.

Obata K, Yamanaka H, Fukuoka T, Yi D, Tokunaga A, Hashimoto N, Yoshikawa H, Noguchi K (2003) Contribution of injured and uninjured dorsal root ganglion neurons to pain behavior and the changes in gene expression following chronic constriction injury of the sciatic nerve in rats. Pain 101:65-77.

Olsson Y (1990) Microenvironment of the peripheral nervous system under normal and pathological conditions. Crit Rev Neurobiol 5:265-311.

Piao ZG, Cho IH, Park CK, Hong JP, Choi SY, Lee SJ, Lee S, Park K, Kim JS, Oh SB (2006) Activation of glia and microglial p38 MAPK in medullary dorsal horn contributes to tactile hypersensitivity following trigeminal sensory nerve injury. Pain 121:219-231.

Raghavendra V, Rutkowski MD, DeLeo JA (2002) The role of spinal neuroimmune activation in morphine tolerance/hyperalgesia in neuropathic and sham-operated rats. J Neurosci 22:9980-9989.

Raghavendra V, Tanga F, DeLeo JA (2003) Inhibition of microglial activation attenuates the development but not existing hypersensitivity in a rat model of neuropathy. J Pharmacol Exp Ther 306:624-630.

Roy A, Fung YK, Liu X, Pahan K (2006) Up-regulation of microglial CD11b expression by nitric oxide. J Biol Chem 281:14971-14980.

Salimi K, Humpel C (2002) Down-regulation of complement receptor 3 and major histocompatibility complex I and II antigen-like immunoreactivity accompanies ramification in isolated rat microglia. Brain Res 946:283-289.

Sallmann S, Jüttler E, Prinz S, Petersen N, Knopf U, Weiser T, Schwaninger M (2000) Induction of interleukin-6 by depolarization of neurons. J Neurosci 20:8637-8642.

Scholz J, Woolf CJ (2007) The neuropathic pain triad: neurons, immune cells and glia. Nat Neurosci 10:1361-1368.

Shortland PJ, Baytug B, Krzyzanowska A, McMahon SB, Priestley JV, Averill S (2006) ATF3 expression in L4 dorsal root ganglion neurons after L5 spinal nerve transection. Eur J Neurosci 23:365-373.

Sweitzer SM, Schubert P, DeLeo JA (2001) Propentofylline, a glial modulating agent, exhibits antiallodynic properties in a rat model of neuropathic pain. J Pharmacol Exp Ther 297:1210-1217.

Tal M, Devor M (1992) Ectopic discharge in injured nerves: comparison of trigeminal and somatic afferents. Brain Res 579:148-151.

Thier M, März P, Otten U, Weis J, Rose-John S (1999) Interleukin-6 (IL-6) 
and its soluble receptor support survival of sensory neurons. J Neurosci Res 55:411-422.

Tikka TM, Koistinaho JE (2001) Minocycline provides neuroprotection against $N$-methyl-D-aspartate neurotoxicity by inhibiting microglia. J Immunol 166:7527-7533.

Tsujino H, Kondo E, Fukuoka T, Dai Y, Tokunaga A, Miki K, Yonenobu K, Ochi T, Noguchi K (2000) Activating transcription factor 3 (ATF3) induction by axotomy in sensory and motoneurons: A novel neuronal marker of nerve injury. Mol Cell Neurosci 15:170-182.

Tsuzuki K, Fukuoka T, Sakagami M, Noguchi K (2003) Increase of preprotachykinin mRNA in the uninjured mandibular neurons after rat infraorbital nerve transection. Neurosci Lett 345:57-60.

Verge GM, Milligan ED, Maier SF, Watkins LR, Naeve GS, Foster AC (2004) Fractalkine (CX3CL1) and fractalkine receptor (CX3CR1) distribution in spinal cord and dorsal root ganglia under basal and neuropathic pain conditions. Eur J Neurosci 20:1150-1160.
Vos BP, Strassman AM, Maciewicz RJ (1994) Behavioral evidence of trigeminal neuropathic pain following chronic constriction injury to the rat's infraorbital nerve. J Neurosci 14:2708-2723.

Watkins LR, Milligan ED, Maier SF (2001) Spinal cord glia: new players in pain. Pain 93:201-205.

Young R (1977) Fiber spectrum of the trigeminal sensory root of frog, cat and man determined by electronmicroscopy. In: Pain in the trigeminal region (Anderson DJ, Matthews B, eds), pp 137-147. Amsterdam: Elsevier.

Yrjänheikki J, Tikka T, Keinänen R, Goldsteins G, Chan PH, Koistinaho J (1999) A tetracycline derivative, minocycline, reduces inflammation and protects against focal cerebral ischemia with a wide therapeutic window. Proc Natl Acad Sci USA 96:13496-13500. 